\title{
Linear Interference Cancellation for the Cell-Free C-RAN Uplink
}

\author{
Reza Mosayebi, Member, IEEE, Mohammad M. Mojahedian, Member, IEEE, \\ and Angel Lozano, Fellow, IEEE
}

\begin{abstract}
Drawing on the notion of parallel interference cancellation, this paper formulates a one-shot linear receiver for the uplink of centralized, possibly cloud-based, radio access networks (C-RANs) operating in a cell-free fashion. This receiver exhibits substantial interference rejection abilities, yet it does not involve any matrix inversions; rather, its structure hinges on the pairwise projections of the users' channel vectors. Its performance is markedly superior to that of matched-filter beamforming, while the computational cost is decidedly inferior to that of an MMSE filter, altogether constituting an attractive alternative in terms of performance vs cost. Furthermore, with a proper sparsification of the channel matrix that it estimates and processes, the proposed receiver can be rendered scalable in the sense of its computational cost per access point not growing with size of the network. Uplink power control is also readily accommodated.
\end{abstract}

Index Terms-C-RAN, cell-free networks, ultradense networks, parallel interference cancellation, power control

\section{INTRODUCTION}

Wireless networks have long been organized under the cellular paradigm, whereby every user is served by a single access point (AP) while being regarded as interference by other APs. Capitalizing on the invariance with the cell size of the signal-to-interference ratio (SIR), such networks have sustainedly increased their area capacity by means of radical densification [?], always within the confines of the cellular paradigm and with only limited forays beyond it [?], [?], [?].

The process of densification is bound to reach a point where the SIR invariance breaks down because of line-ofsight propagation across cells and an ensuing surge in othercell interference [?]. In conjunction with the interest in extending to the radio access the ongoing transformation to software-defined networks, this motivates the new paradigm of centralized, possibly cloud-based, radio access networks (CRANs) [?], [?], [?], [?], [?], [?], [?], [?], [?]. In a C-RAN, the AP receivers consist solely of antennas, amplifiers, and downconverters, connected by powerful fronthaul lines to an edge datacenter where the baseband processing resides.

C-RANs are compatible with a cellular structure, but they also open the door to more general cell-free structures where every user is potentially served by every AP, inheriting and taking to their limit the principles of cell cooperation [?], [?], [?], [?]. Influenced by massive MIMO, much of the

The authors are with Univ. Pompeu Fabra, 08018 Barcelona (e-mail: \{reza.mosayebi, mohammadmahdi.mojahedian, angel.lozano\}@upf.edu) and their work is supported by the European Research Council under the H2020 Framework Programme/ERC grant agreement 694974, by MINECO's Projects RTI2018-102112 and RTI2018-101040, and by the ICREA academia program. Parts of this paper were presented at the 2020 Asilomar Conference [?]. growing literature on cell-free networks postulates matchedfilter (MF) beamformers, which may advantageously admit somewhat distributed downlink implementations [?], [?], [?], [?], [?], [?], [?], [?], [?], [?], [?], [?], [?], [?], [?], [?], [?], [?]. However, the ensuing performance compares only modestly with that of a cellular network. The true potential of C-RANs emerges when their centralized nature is exploited to feature more sophisticated transmitters and receivers that take interference into account. In the uplink specifically, a linear minimum mean-square error (MMSE) receiver would perform decidedly better than its MF counterpart [?], [?], [?], [?], being in fact the optimum linear receiver in terms of SINR and spectral efficiency, but at the cost of having to invert a large-dimensional matrix on each fading coherence block (every few ms and every few hundred $\mathrm{kHz}$ ). The performance and computational disparity between these linear receiver extremes, the basic MF and the optimum MMSE, invites exploring alternatives that can outperform the former without the computational cost of the latter.

Since the MF is optimum against the noise, any attempt to improve upon it necessarily entails dealing with the interference. And, since the computational cost of an MMSE filter is dominated by the need for matrix inversions, reducing that cost calls for a receiver whose computation is devoid of inverses. Combing both requisites, our stated goal becomes that of an interference-aware inverse-free receiver, and a promising path towards this objective lies in the realm of interference cancellation.

The most established interference cancellation technique in MIMO communication is successive interference cancellation (SIC), which in fact serves as building block to an informationtheoretically optimum structure [?, sec. 5.8]. While therefore appealing in small-dimensional MIMO settings, however, SIC is out of the question for a C-RAN with hundreds or even thousands of users, as an enormous number of iterations would be needed to successively peel off all those users. A parallel interference cancellation (PIC) approach is much more enticing, as the number of iterations therein can be decoupled from the number of users, and further because PIC schemes are naturally amenable to parallelization. There is an extensive volume of research on PIC receivers that can be built upon, both without [?], [?], [?], [?], [?], [?], [?], [?], [?], [?], [?], [?], [?], [?], [?] and with [?], [?], [?], [?], [?] the channel decoders in the cancellation loops. Although having the decoders in the PIC loops promises superior performance, the large number of users - again — and their potential asynchronicity, especially in the face of independent hybrid ARQ processing, strongly 
suggests applying PIC before the decoding. Also, while postdecoding PIC would necessarily be nonlinear, pre-decoding PIC has the added flexibility of admitting both linear and nonlinear interference estimators, and this is the option we concentrate on. Precisely, our starting point is a soft PIC architecture featuring linear interference estimators, which advantageously do not depend on the signal distribution. The main contributions are then as follows.

- The one-shot linear receiver that arises from a single stage of linear PIC is formulated for the cell-free setting, as an alternative to the MF and MMSE structures. As part of this formulation, a new and readily computable weighting is derived for the interference estimates. The obtained receiver performs close to the MMSE baseline with only a fraction of its computational cost and, in particular, with no need for matrix inversions. It also outperforms firstorder polynomial expansions of that MMSE baseline.

- By estimating and processing a sparsified version of the channel matrix in lieu of the actual one, the proposed receiver is rendered scalable in the sense of its computational cost per AP not growing with the network size. With a small subset of APs receiving each user, and a small subset of users being processed by each AP, the original unsparsified performance is essentially matched; this is consistent with other works on the reach that is necessary for user-centric communication to perform satisfactorily [?], [?], [?], [?], [?], [?].

- Power control, a very important ingredient in uplink transmission, is also incorporated into the formulation and shown to be fully compatible with the advocated receiver architecture.

The performance evaluation in the paper befits from recent results showing that, under the premise of AP positions that are agnostic to the radio propagation, shadow fading renders an arbitrary network approximately Poisson-like from the vantage of any user [?], [?]. This approximation sharpens as the shadowing strengthens, being precise for values of interest [?], [?], [?], [?]. Relying on these findings, the performance is evaluated with APs and users positioned uniformly at random, with shadow fading implicitly embedded in the geometry.

The manuscript is organized as follows. Sections ?? and ?? introduce the models and signals employed throughout, and Section ?? lays down the two basic benchmarks, namely the MF and the MMSE structures. In Section ??, the proposed linear PIC receiver is put forth, and its performance and cost are evaluated relative to the benchmarks. The issue of scalability, all-important in cell-free networks, is discussed in Section ??, which sets the stage for a sparse and scalable version of the PIC receiver in Section ??. Finally, Section ?? brings power control into the picture and Section ?? concludes the paper.

Notation: Vectors and matrices are represented by lowercase and uppercase boldface symbols, respectively. The expectation operator is denoted by $\mathbb{E}[\cdot]$ while $\propto$ indicates proportionality and $\mathcal{N}_{\mathbb{C}}\left(m, \sigma^{2}\right)$ denotes a circularly symmetric complex Gaussian scalar distribution with mean $m$ and variance $\sigma^{2}$.

\section{NETWORK AND CHANNEL MODELS}

The networks under consideration feature $N$ APs and $K$ users, all equipped with a single omnidirectional antenna. Some time-frequency resource units are reserved for pilot transmissions from the users, based on which the channels are estimated by the APs. The remaining resource units are available for data transmission.

\section{A. Large-scale Modeling}

The local-average channel gain between the $k$ th user and the $n$th AP, denoted by $G_{n, k}$, subsumes distance-dependent pathloss and shadowing. For the pathloss, we consider two distinct models. First, the commonplace single-slope model

$$
G_{n, k}=\operatorname{dist}_{n, k}^{-\eta}
$$

where dist ${ }_{n, k}$ is the distance between the $k$ th user and the $n$th AP. Second, the dual-slope model

$$
G_{n, k}= \begin{cases}\text { dist }_{n, k}^{-2} & \text { dist }_{n, k} \leq \text { breakpoint } \\ \text { breakpoint }^{\eta-2} \text { dist }_{n, k}^{-\eta} & \text { dist }_{n, k}>\text { breakpoint }^{-\eta}\end{cases}
$$

The dual-slope model entails the additional parametrization of the breakpoint distance, but it better reflects the idiosyncrasy of the radio propagation in ultradense networks.

Letting $P$ denote a user's transmit power, and with $\sigma^{2}$ the noise power, the local-average SNR of user $k$ at the $n$th AP is

$$
\mathrm{SNR}_{n, k}=G_{n, k} \frac{P}{\sigma^{2}}
$$

The large-scale gains, and thus the local-average SNRs, are known and stable over each network snapshot.

\section{B. Small-scale Modeling}

Besides $G_{n, k}$, the channel between the $k$ th user and the $n$th AP features a small-scale fading coefficient $h_{n, k} \sim \mathcal{N}_{\mathbb{C}}(0,1)$, independent across users and APs.

\section{Simulation Environment}

For the purpose of performance evaluation, we resort to a wrapped-around universe with $N=200$ APs (elevated $2 \mathrm{~m}$ above the users to avoid distance singularities). The AP and user positions are drawn uniformly at random. Thanks to the wrap-around, all of them contribute equally to the statistics. The number of network snapshots is adjusted, depending on $K$, to push the $95 \%$ confidence interval below $0.05 \mathrm{~dB}$.

Unless otherwise stated, $P / \sigma^{2}$ is such that $\mathrm{SNR}_{n, k}=10 \mathrm{~dB}$ for dist $t_{n, k}=d$, where $d$ would be spacing among APs if the network were arranged as a hexagonal grid with the same spatial density that our network. Under reasonable values for the transmit power, bandwidth, and pathloss intercept [?], this is compatible with ultradense deployments $(d \approx 5-20 \mathrm{~m})$ and in fact conservatively low.

In the dual-slope pathloss model, $P / \sigma^{2}$ is adjusted such that the SNR at the breakpoint distance $d$ is preserved. 


\section{Channel Estimation and Data Transmission}

Disregarding pilot contamination for now, the MMSE channel estimate $\hat{h}_{n, k}$ gathered by the network upon observation at the $n$th $\mathrm{AP}$ of a pilot transmission from user $k$ satisfies $h_{n, k}=\hat{h}_{n, k}+\tilde{h}_{n, k}$ where

$$
\mathbb{E}\left[\left|\hat{h}_{n, k}\right|^{2}\right]=\frac{\mathrm{SNR}_{n, k}}{1+\mathrm{SNR}_{n, k}},
$$

while

$$
\tilde{h}_{n, k} \sim \mathcal{N}_{\mathbb{C}}\left(0, \frac{1}{1+\mathrm{SNR}_{n, k}}\right)
$$

is uncorrelated error. Generalizing (??)-(??) to multipleas opposed to one-pilot transmissions per user and per fading coherence block, or to power-boosted pilots, entails a straightforward scaling of $\mathrm{SNR}_{n, k}$ therein [?]. Ultimately, the overhead corresponding to the resource units consumed by pilots must be discounted from the gross spectral efficiency.

Subsequently, upon data transmission, on a given timefrequency resource unit the $n$th AP observes

$$
\begin{aligned}
y_{n} & =\sum_{k=0}^{K-1} \sqrt{G_{n, k}} h_{n, k} x_{k}+v_{n} \\
& =\sum_{k=0}^{K-1} \sqrt{G_{n, k}} \hat{h}_{n, k} x_{k}+\underbrace{\sum_{k=0}^{K-1} \sqrt{G_{n, k}} \tilde{h}_{n, k} x_{k}+v_{n}}_{z_{n}},
\end{aligned}
$$

where $x_{k}$ is the signal from user $k$, satisfying $\mathbb{E}\left[\left|x_{k}\right|^{2}\right]=P$, while $v_{n} \sim \mathcal{N}_{\mathbb{C}}\left(0, \sigma^{2}\right)$.

The transmit-receive relationship between users and APs can be vectorized into

$$
\begin{aligned}
\boldsymbol{y}=\left[\begin{array}{c}
y_{0} \\
\vdots \\
y_{N-1}
\end{array}\right] & =\sum_{k=0}^{K-1} \hat{\boldsymbol{c}}_{k} x_{k}+\boldsymbol{z} \\
& =\hat{\boldsymbol{C}} \boldsymbol{x}+\boldsymbol{z},
\end{aligned}
$$

where $\hat{\boldsymbol{C}}=\left[\hat{\boldsymbol{c}}_{0} \cdots \hat{\boldsymbol{c}}_{K-1}\right]$ with

$$
\hat{\boldsymbol{c}}_{k}=\left[\begin{array}{c}
\sqrt{G_{0, k}} \hat{h}_{0, k} \\
\vdots \\
\sqrt{G_{N-1, k}} \hat{h}_{N-1, k}
\end{array}\right],
$$

while

$$
\boldsymbol{z}=\left[\begin{array}{c}
z_{0} \\
\vdots \\
z_{N-1}
\end{array}\right], \quad \boldsymbol{x}=\left[\begin{array}{c}
x_{0} \\
\vdots \\
x_{K-1}
\end{array}\right],
$$

and $\mathbb{E}\left[\boldsymbol{z} \boldsymbol{z}^{*}\right]=\sigma^{2} \boldsymbol{D}$ with $\boldsymbol{D}$ an $N \times N$ matrix whose $(n, n)$ th entry is

$$
[\boldsymbol{D}]_{n, n}=1+\sum_{k=0}^{K-1} \frac{\mathrm{SNR}_{n, k}}{1+\mathrm{SNR}_{n, k}},
$$

and whose off-diagonal entries are zero on account of the independence of the small-scale fading and noise across APs.

\section{Network-Wide Receiver Benchmarks}

\section{A. $M F$}

With the channel estimates taken to be correct, the MF receiver for all $K$ users, or more precisely the whitening MF, is given by the matrix $D^{-1} \hat{C}$. For given fading realizations, this receiver delivers

$$
\begin{aligned}
\operatorname{sinr}_{k}^{\mathrm{MF}} & =\frac{\mathbb{E}\left[\left|\hat{\boldsymbol{c}}_{k}^{*} \boldsymbol{D}^{-1} \hat{\boldsymbol{c}}_{k} x_{k}\right|^{2} \mid \hat{\boldsymbol{c}}_{k}\right]}{\sum_{\ell \neq k} \mathbb{E}\left[\left|\hat{\boldsymbol{c}}_{k}^{*} \boldsymbol{D}^{-1} \hat{\boldsymbol{c}}_{\ell} x_{\ell}+\hat{\boldsymbol{c}}_{k}^{*} \boldsymbol{D}^{-1} \boldsymbol{z}\right|^{2} \mid \hat{\boldsymbol{c}}_{k}, \hat{\boldsymbol{c}}_{\ell}\right]} \\
& =\frac{\left|\hat{\boldsymbol{c}}_{k}^{*} \boldsymbol{D}^{-1} \hat{\boldsymbol{c}}_{k}\right|^{2}}{\sum_{\ell \neq k}\left|\hat{\boldsymbol{c}}_{k}^{*} \boldsymbol{D}^{-1} \hat{\boldsymbol{c}}_{\ell}\right|^{2}+\frac{\sigma^{2}}{P} \hat{\boldsymbol{c}}_{k}^{*} \boldsymbol{D}^{-1} \hat{\boldsymbol{c}}_{k}} .
\end{aligned}
$$

\section{B. MMSE}

Based on the available channel estimates, the MMSE receiver for all $K$ users is [?, sec. 6.4]

$$
\begin{aligned}
\boldsymbol{W}^{\mathrm{MMSE}} & =\left(\hat{\boldsymbol{C}} \hat{\boldsymbol{C}}^{*}+\frac{\sigma^{2}}{P} \boldsymbol{D}\right)^{-1} \hat{\boldsymbol{C}} \\
& =\boldsymbol{D}^{-1} \hat{\boldsymbol{C}}\left(\hat{\boldsymbol{C}}^{*} \boldsymbol{D}^{-1} \hat{\boldsymbol{C}}+\frac{\sigma^{2}}{P} \boldsymbol{I}\right)^{-1}
\end{aligned}
$$

which gives

$$
\operatorname{sinr}_{k}^{\mathrm{MMSE}}=\frac{\left|\boldsymbol{w}_{k}^{\mathrm{MMSE} *} \hat{\boldsymbol{c}}_{k}\right|^{2}}{\sum_{\ell \neq k}\left|\boldsymbol{w}_{k}^{\mathrm{MMSE} *} \hat{\boldsymbol{c}}_{\ell}\right|^{2}+\frac{\sigma^{2}}{P} \boldsymbol{w}_{k}^{\mathrm{MMSE} *} \boldsymbol{D} \boldsymbol{w}_{k}^{\mathrm{MMSE}}},
$$

where

$$
\begin{aligned}
\boldsymbol{w}_{k}^{\mathrm{MMSE}} & =\left[\boldsymbol{W}^{\mathrm{MMSE}}\right]_{:, k} \\
& =\left(\hat{\boldsymbol{C}} \hat{\boldsymbol{C}}^{*}+\frac{\sigma^{2}}{P} \boldsymbol{D}\right)^{-1} \hat{\boldsymbol{c}}_{k}
\end{aligned}
$$

is the $k$ th column of $\boldsymbol{W}^{\mathrm{MMSE}}$. With a bit of algebra, it can be seen that $[?$, sec. 6.4$]$

$$
\operatorname{sinr}_{k}^{\mathrm{MMSE}}=\frac{\hat{\boldsymbol{c}}_{k}^{*}\left(\hat{\boldsymbol{C}} \hat{\boldsymbol{C}}^{*}+\frac{\sigma^{2}}{P} \boldsymbol{D}\right)^{-1} \hat{\boldsymbol{c}}_{k}}{1-\hat{\boldsymbol{c}}_{k}^{*}\left(\hat{\boldsymbol{C}} \hat{\boldsymbol{C}}^{*}+\frac{\sigma^{2}}{P} \boldsymbol{D}\right)^{-1} \hat{\boldsymbol{c}}_{k}} .
$$

\section{LiNEAR RECEIVER BASED ON SOFT PIC}

\section{A. Soft PIC}

From the standpoint of user $k$, (??) can be rewritten as

$$
\boldsymbol{y}=\hat{\boldsymbol{c}}_{k} x_{k}+\underbrace{\sum_{\ell \neq k} \hat{\boldsymbol{c}}_{\ell} x_{\ell}}_{\text {Interference }}+\boldsymbol{z} .
$$

The PIC proposition (see Fig. ??) is to subtract a soft estimate of the interference from $y$ so as to obtain

$$
\boldsymbol{y}_{k}=\boldsymbol{y}-\sum_{\ell \neq k} \hat{\boldsymbol{c}}_{\ell} \hat{x}_{\ell}
$$

where $\hat{x}_{\ell}$ is a soft estimate of $x_{\ell}$. From $\boldsymbol{y}_{k}$, a better decision statistic can be derived for user $k$ than would be obtained directly from $\boldsymbol{y}$. Any estimation strategy, linear or nonlinear, can in principle be applied to obtain $\hat{x}_{0}, \ldots, \hat{x}_{K-1}$. 


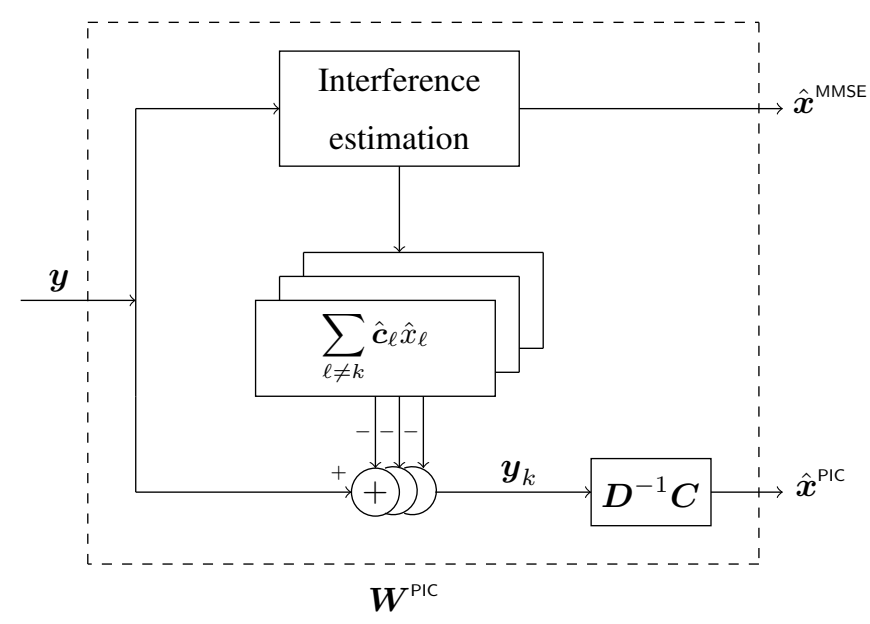

Fig. 1. Block diagram of a PIC receiver, including the sub-blocks handling interference estimation, cancellation, and whitening MF.

\section{B. Linear Interference Estimation}

Linear estimators have the advantage of not depending on the signal distributions, but only on covariances, a point that endows the receiver with broader generality. Moreover, when the interference estimators are linear, the overall receiver becomes itself linear [?], [?], [?], [?], [?], [?], [?], [?]. Adopting therefore a linear interference estimation strategy,

$$
\hat{x}_{\ell}=\boldsymbol{a}_{\ell}^{*} \boldsymbol{y}
$$

such that (??) can be rewritten as

$$
\begin{aligned}
\boldsymbol{y}_{k} & =\boldsymbol{y}-\sum_{\ell \neq k} \hat{\boldsymbol{c}}_{\ell} \boldsymbol{a}_{\ell}^{*} \boldsymbol{y} \\
& =\left(\boldsymbol{I}-\sum_{\ell \neq k} \hat{\boldsymbol{c}}_{\ell} \boldsymbol{a}_{\ell}^{*}\right) \boldsymbol{y}
\end{aligned}
$$

and, further applying a whitening MF to $\boldsymbol{y}_{k}$, the decision statistic for user $k$ becomes

$$
\hat{\boldsymbol{c}}_{k}^{*} \boldsymbol{D}^{-1} \boldsymbol{y}_{k}=\underbrace{\left(\hat{\boldsymbol{c}}_{k}^{*} \boldsymbol{D}^{-1}-\sum_{\ell \neq k} \hat{\boldsymbol{c}}_{k}^{*} \boldsymbol{D}^{-1} \hat{\boldsymbol{c}}_{\ell} \boldsymbol{a}_{\ell}^{*}\right)}_{\boldsymbol{w}_{k}^{\mathrm{PC} *}} \boldsymbol{y} .
$$

The single-stage PIC architecture is thus equivalent to the linear receiver

$$
\boldsymbol{w}_{k}^{\mathrm{PIC}}=\boldsymbol{D}^{-1} \hat{\boldsymbol{c}}_{k}-\sum_{\ell \neq k} \boldsymbol{a}_{\ell} \hat{\boldsymbol{c}}_{\ell}^{*} \boldsymbol{D}^{-1} \hat{\boldsymbol{c}}_{k} \quad k=0, \ldots, K-1,
$$

which is one-shot, meaning that it operates on $\boldsymbol{y}$ only once, and with a performance-cost tradeoff that depends on the interference estimators $\boldsymbol{a}_{0}, \ldots, \boldsymbol{a}_{K-1}$.

An inviting choice for $\boldsymbol{a}_{\ell}$ is the linear MMSE estimator

$$
\boldsymbol{a}_{\ell}=\left(\mathbb{E}\left[\boldsymbol{y} \boldsymbol{y}^{*} \mid \hat{\boldsymbol{c}}_{0}, \ldots, \hat{\boldsymbol{c}}_{K-1}\right]\right)^{-1} \mathbb{E}\left[\boldsymbol{y} x_{\ell}^{*} \mid \hat{\boldsymbol{c}}_{0}, \ldots, \hat{\boldsymbol{c}}_{K-1}\right],
$$

which operates on $\boldsymbol{y}$ and with the knowledge of $\hat{\boldsymbol{c}}_{0}, \ldots, \hat{\boldsymbol{c}}_{K-1}$. Not surprisingly, the above returns $\boldsymbol{a}_{\ell}=\boldsymbol{w}_{\ell}^{\mathrm{MMSE}}$, the same filter employed by network-wise MMSE reception to recover user $k$. Thus, as indicated in Fig. ??, the interference estimates would then coincide with the MMSE receiver output, whose
SINR cannot be improved upon by any linear receiver. Indeed, plugging (??) into (??), what emerges after some algebra (see Appendix ??) is

$$
\boldsymbol{w}_{k}^{\mathrm{PIC}} \propto \boldsymbol{w}_{k}^{\mathrm{MMSE}}
$$

with the scaling factor not affecting the SINR. This confirms that the one-shot linear receiver that results from applying a PIC stage with MMSE interference estimation is itself equivalent to a linear MMSE receiver, motivating the exploration of other linear interference estimators.

\section{Scalar Linear Interference Estimation}

A way to circumvent the need for matrix inversions is to estimate each interference term on the basis of a scalar input, rather than from $\boldsymbol{y}$, and a prime candidate is the output of the respective MF. Indeed, the classic linear PIC formulation [?], applied to our setting, would rely on

$$
\begin{aligned}
\hat{x}_{\ell} & =\frac{1}{\hat{\boldsymbol{c}}_{\ell}^{*} \boldsymbol{D}^{-1} \hat{\boldsymbol{c}}_{\ell}} \hat{\boldsymbol{c}}_{\ell}^{*} \boldsymbol{D}^{-1} \boldsymbol{y} \\
& =x_{\ell}+\boldsymbol{z}_{\ell},
\end{aligned}
$$

where $z_{\ell}$ contains noise plus interference. This formulation, suported by the analogy between multistage linear PIC on one hand and the Jacobi method for systems of equations on the other [?], tends to be effective for receivers with many stages (ideally infinitely many), although it may at times exhibit oscillating or even diverging behaviors. By applying a dampening weight at each PIC stage, these behaviors can be avoided and the convergence accelerated [?]. Existing forms for these dampening weights, however, were largely developed in the context of CDMA multistage receivers and do not match our needs; they amount to a common weight for all users at each stage, with these weights often the result of a computationally intensive task. Rather, we seek PIC weights that are:

1) Intended for a single stage of PIC.

2) Individualized for each user, so as to represent the disparity of channel conditions across a cell-free network.

3) Readily computable.

Departing from previous strategies where the weights were suitably optimized for some post-PIC receiver metric (say the mean-square error [?] or the error probability [?]) over a number of stages, we set the weights so as to optimize the estimation of the interference before the PIC. To that end, let us return to (??) and reformulate it on the basis of the corresponding MF output, i.e.,

$$
\hat{x}_{\ell}=a_{\ell}^{*} \hat{\boldsymbol{c}}_{\ell}^{*} \boldsymbol{D}^{-1} \boldsymbol{y} .
$$

Then, setting the estimation coefficient $a_{\ell}$ so as to render $\hat{x}_{\ell}$ the linear MMSE estimate of $x_{\ell}$ from $\hat{\boldsymbol{c}}_{\ell}^{*} \boldsymbol{D}^{-1} \boldsymbol{y}$ given the knowledge of $\hat{\boldsymbol{c}}_{0}, \ldots, \hat{\boldsymbol{c}}_{K-1}$, we obtain

$$
a_{\ell}=\frac{\mathbb{E}\left[\hat{\boldsymbol{c}}_{\ell}^{*} \boldsymbol{D}^{-1} \boldsymbol{y} \cdot x_{\ell}^{*} \mid \hat{\boldsymbol{c}}_{0}, \ldots, \hat{\boldsymbol{c}}_{K-1}\right]}{\mathbb{E}\left[\hat{\boldsymbol{c}}_{\ell}^{*} \boldsymbol{D}^{-1} \boldsymbol{y} \cdot \boldsymbol{y}^{*} \boldsymbol{D}^{-1} \hat{\boldsymbol{c}}_{\ell} \mid \hat{\boldsymbol{c}}_{0}, \ldots, \hat{\boldsymbol{c}}_{K-1}\right]}
$$




$$
\begin{aligned}
& =\frac{\mathbb{E}\left[\hat{\boldsymbol{c}}_{\ell}^{*} \boldsymbol{D}^{-1}\left(\sum_{k=0}^{K-1} \hat{\boldsymbol{c}}_{k} x_{k}+\boldsymbol{z}\right) x_{\ell}^{*} \mid \hat{\boldsymbol{c}}_{0}, \ldots, \hat{\boldsymbol{c}}_{K-1}\right]}{\mathbb{E}\left[\hat{\boldsymbol{c}}_{\ell}^{*} \boldsymbol{D}^{-1}\left(\sum_{j=0}^{K-1} \hat{\boldsymbol{c}}_{j} x_{j}+\boldsymbol{z}\right)\left(\sum_{i=0}^{K-1} \hat{\boldsymbol{c}}_{i} x_{i}+\boldsymbol{z}\right)^{*} \boldsymbol{D}^{-1} \hat{\boldsymbol{c}}_{\ell} \mid \hat{\boldsymbol{c}}_{0}, \ldots, \hat{\boldsymbol{c}}_{K-1}\right]} \\
& =\frac{\hat{\boldsymbol{c}}_{\ell}^{*} \boldsymbol{D}^{-1} \sum_{k=0}^{K-1} \hat{\boldsymbol{c}}_{k} \mathbb{E}\left[x_{k} x_{\ell}^{*}\right]}{\hat{\boldsymbol{c}}_{\ell}^{*} \boldsymbol{D}^{-1} \sum_{j=0}^{K-1} \hat{\boldsymbol{c}}_{j} \sum_{i=0}^{K-1} \mathbb{E}\left[x_{j} x_{i}^{*}\right] \hat{\boldsymbol{c}}_{i}^{*} \boldsymbol{D}^{-1} \hat{\boldsymbol{c}}_{\ell}+\hat{\boldsymbol{c}}_{\ell}^{*} \boldsymbol{D}^{-1} \mathbb{E}\left[\boldsymbol{z} \boldsymbol{z}^{*}\right] \boldsymbol{D}^{-1} \hat{\boldsymbol{c}}_{\ell}} \\
& =\frac{\hat{\boldsymbol{c}}_{\ell}^{*} \boldsymbol{D}^{-1} \hat{\boldsymbol{c}}_{\ell}}{\sum_{j=0}^{K-1}\left|\hat{\boldsymbol{c}}_{\ell}^{*} \boldsymbol{D}^{-1} \hat{\boldsymbol{c}}_{j}\right|^{2}+\frac{\sigma^{2}}{P} \hat{\boldsymbol{c}}_{\ell}^{*} \boldsymbol{D}^{-1} \hat{\boldsymbol{c}}_{\ell}} .
\end{aligned}
$$

Recalling (??), and after a bit of algebra, the above can be seen to equal

$$
a_{\ell}=\frac{\sin r_{\ell}^{\mathrm{MF}}}{1+\operatorname{sinr}_{\ell}^{\mathrm{MF}}} \frac{1}{\hat{\boldsymbol{c}}_{\ell}^{*} \boldsymbol{D}^{-1} \hat{\boldsymbol{c}}_{\ell}}
$$

such that

$$
\hat{x}_{\ell}=\frac{\operatorname{sinr}_{\ell}^{\mathrm{MF}}}{1+\operatorname{sinr}_{\ell}^{\mathrm{MF}}} \frac{1}{\hat{\boldsymbol{c}}_{\ell}^{*} \boldsymbol{D}^{-1} \hat{\boldsymbol{c}}_{\ell}} \hat{\boldsymbol{c}}_{\ell}^{*} \boldsymbol{D}^{-1} \boldsymbol{y} .
$$

Contrasting (??) with (??), the new element in the former is seen to be the weight

$$
\frac{\operatorname{sinr}_{\ell}^{\mathrm{MF}}}{1+\sin r_{\ell}^{\mathrm{MF}}}
$$

which is pleasing in the sense of:

1) Yielding, for the single PIC stage under consideration and on the basis of the MF outputs, the MMSE interference estimates.

2) Being individualized as a function of each user's SINR prior to the PIC.

3) Being easily computable from only such SINR, which is a quantity both relevant and available.

From (??), the one-shot linear PIC receiver becomes

$$
\boldsymbol{w}_{k}^{\mathrm{PIC}}=\boldsymbol{D}^{-1} \hat{\boldsymbol{c}}_{k}-\sum_{\ell \neq k} \frac{\operatorname{sinr} r_{\ell}^{\mathrm{MF}}}{1+\operatorname{sinr}_{\ell}^{\mathrm{MF}}} \frac{\hat{\boldsymbol{c}}_{\ell}^{*} \boldsymbol{D}^{-1} \hat{\boldsymbol{c}}_{\ell}}{\hat{\boldsymbol{c}}_{\ell}^{*} \boldsymbol{D}^{-1} \hat{\boldsymbol{c}}_{\ell}} \boldsymbol{D}^{-1} \hat{\boldsymbol{c}}_{\ell}
$$

and the SINR of user $k$ at the output of $\boldsymbol{w}_{k}^{\mathrm{PIC}}$ is

$$
\operatorname{sinr}_{k}^{\mathrm{PCC}}=\frac{\left|\boldsymbol{w}_{k}^{\mathrm{PIC} *} \hat{\boldsymbol{c}}_{k}\right|^{2}}{\sum_{\ell \neq k}\left|\boldsymbol{w}_{k}^{\mathrm{PIC} *} \hat{\boldsymbol{c}}_{\ell}\right|^{2}+\frac{\sigma^{2}}{P} \boldsymbol{w}_{k}^{\mathrm{PlC} *} \boldsymbol{D} \boldsymbol{w}_{k}^{\mathrm{PIC}}} .
$$

The expression in (??) offers considerable intuition on how the PIC stage modifies the original MF for user $k$ :

- As sinr $\mathrm{MF}_{\ell}^{\mathrm{MF}}$ grows, the confidence on the estimate of the $\ell$ th term increases and a larger share of the ensuing interference is cancelled. Equivalently, $\boldsymbol{w}_{k}^{\text {PIC }}$ deviates progressively from its MF direction so as to avoid the $\ell$ th interferer.

- As the projection of $\hat{\boldsymbol{c}}_{\ell}$ onto $\hat{\boldsymbol{c}}_{k}$ grows strong, the interference from the $\ell$ th term further afflicts the $k$ th user and the cancellation of the former from the latter intensifies accordingly. Equivalently, $\boldsymbol{w}_{k}^{\mathrm{PIC}}$ again deviates from its MF direction, away from the $\ell$ th interferer.

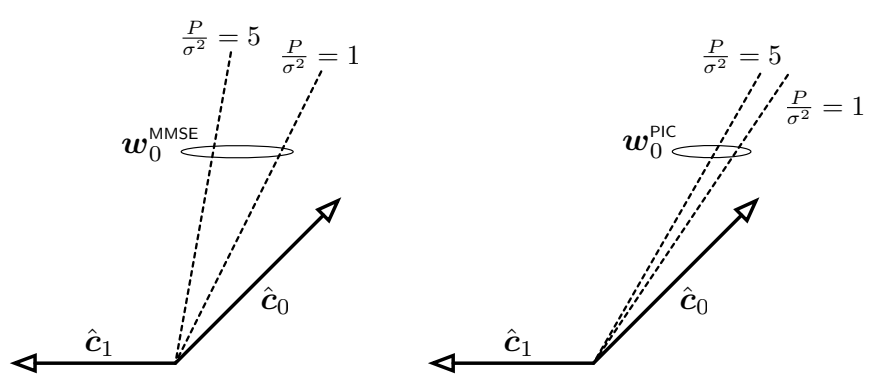

Fig. 2. On the left-hand side, directions taken by $\boldsymbol{w}_{0}^{\mathrm{MMSE}}$ for $\hat{\boldsymbol{c}}_{0}=\left[\begin{array}{ll}1 & 1\end{array}\right]^{\mathrm{T}}$ and $\hat{\boldsymbol{c}}_{1}=\left[\begin{array}{ll}-1 & 0\end{array}\right]^{\mathrm{T}}$ with $P / \sigma^{2}=1$ and $P / \sigma^{2}=5$. On the right-hand side, respective directions for $\boldsymbol{w}_{0}^{\mathrm{PIC}}$. In all cases, $\boldsymbol{D}=\boldsymbol{I}$.

Altogether, each initial MF vector is modified so as to reduce its projection on the rest, a procedure that is somewhat akin to what unfolds with MMSE reception and that can be illustrated in a toy setting with $K=N=2$. Consider

$$
\hat{\boldsymbol{c}}_{0}=\left[\begin{array}{l}
1 \\
1
\end{array}\right], \quad \hat{\boldsymbol{c}}_{1}=\left[\begin{array}{c}
-1 \\
0
\end{array}\right]
$$

with $\boldsymbol{D}=\boldsymbol{I}$. The MF receiver for user 0 would point on the direction of $\hat{\boldsymbol{c}}_{0}$ whereas, as shown on the left-hand side of Fig. ??, the corresponding MMSE receiver tilts to be more orthogonal to $\hat{\boldsymbol{c}}_{1}$; moderately for $P / \sigma^{2}=1$, and more pronouncedly for $P / \sigma^{2}=5$. The respective PIC receiver directions for user 0 are depicted on the right-hand side of the figure; they also lean to increase their orthogonality to $\hat{c}_{1}$, but somewhat short of the optimum inclination. This reflects the essential difference between both receiver types, namely the amount of information based on which they align their receive vectors: finding the SINR-maximizing directions (left-hand side of the figure) requires processing $\hat{C}$ jointly, and inevitably a matrix inversion, whereas the PIC approximation to those directions (right-hand side) only necessitates the pairwise projection of its columns, $\hat{\boldsymbol{c}}_{0}, \ldots, \hat{\boldsymbol{c}}_{K-1}$. This approximation errs on the side of conservatism, i.e., of some shortage in interference rejection rather than a loss in intended signal.

An alternative perspective on how the MF for user $k$ is altered by the PIC procedure can be offered by rewriting (??) as

$\boldsymbol{w}_{k}^{\mathrm{PLC}}=\boldsymbol{D}^{-1 / 2}\left\|\boldsymbol{D}^{-1 / 2} \hat{\boldsymbol{c}}_{k}\right\|\left(\boldsymbol{e}_{k}-\sum_{\ell \neq k} \frac{\operatorname{sinr}_{\ell}^{\mathrm{MF}}}{1+\operatorname{sinr}_{\ell}^{\mathrm{MF}}} \cos \theta_{k, \ell} \boldsymbol{e}_{j}\right)$,

where $\theta_{k, \ell}$ is the angle spanned by $\boldsymbol{D}^{-1 / 2} \hat{\boldsymbol{c}}_{k}$ and $\boldsymbol{D}^{-1 / 2} \hat{\boldsymbol{c}}_{\ell}$ while $\boldsymbol{e}_{0}, \ldots, \boldsymbol{e}_{K-1}$ are unit vectors aligned, respectively, with $\boldsymbol{D}^{-1 / 2} \hat{\boldsymbol{c}}_{0}, \ldots, \boldsymbol{D}^{-1 / 2} \hat{\boldsymbol{c}}_{K-1}$. The change in the orientation of $\boldsymbol{w}_{k}^{\mathrm{PIC}}$ with respect to $\boldsymbol{w}_{k}^{\mathrm{MF}}$ is determined by the bracketed term and seen to depend, for the $\ell$ th interferer, on the product of $\cos \theta_{k, \ell}$ and the weight $\operatorname{sinr}_{\ell}^{\mathrm{MF}} /\left(1+\operatorname{sinr}_{\ell}^{\mathrm{MF}}\right)$.

It is interesting to contrast the linear PIC receiver with an existing inverse-free alternative to the MMSE receiver, namely the MMSE truncated polynomial expansion (TPE). Expanding the inverse in (??) around $\boldsymbol{D}$, one obtains [?], [?]

$$
\boldsymbol{w}_{k}^{\text {MMSE }} \propto \boldsymbol{D}^{-1 / 2} \sum_{q=0}^{\infty}\left(\boldsymbol{I}-2 \psi_{k} \boldsymbol{\Sigma}\right)^{q} \boldsymbol{D}^{-1 / 2} \hat{\boldsymbol{c}}_{k}
$$


with convergence assured provided that the coefficient $\psi_{k}$ is within a certain range and where, for the sake of compactness, the shorthand notation $\boldsymbol{\Sigma}=\boldsymbol{D}^{-1 / 2} \hat{\boldsymbol{C}} \hat{\boldsymbol{C}}^{*} \boldsymbol{D}^{-1 / 2}+\frac{\sigma^{2}}{P} \boldsymbol{I}$ was introduced. Truncating the series, polynomial approximations to $\boldsymbol{w}_{k}^{\text {MMSE }}$ are obtained. The zero-order truncation in particular returns the MF while the first-order TPE gives

$$
\boldsymbol{w}_{k}^{\text {TPE }} \propto \boldsymbol{D}^{-1} \hat{\boldsymbol{c}}_{k}-\psi_{k} \boldsymbol{D}^{-1 / 2} \boldsymbol{\Sigma} \boldsymbol{D}^{-1 / 2} \hat{\boldsymbol{c}}_{k}
$$

and the form for $\psi_{k}$ that maximizes the SINR under such truncation is [?]

$$
\psi_{k}=\frac{\hat{\boldsymbol{c}}_{k}^{*} \boldsymbol{D}^{-1 / 2} \boldsymbol{\Sigma}\left(\gamma_{k} \boldsymbol{I}-\boldsymbol{D}^{-1 / 2} \hat{\boldsymbol{c}}_{k} \hat{\boldsymbol{c}}_{k}^{*} \boldsymbol{D}^{-1 / 2}\right) \boldsymbol{\Sigma} \boldsymbol{D}^{-1 / 2} \hat{\boldsymbol{c}}_{k}}{\hat{\boldsymbol{c}}_{k}^{*} \boldsymbol{D}^{-1 / 2} \boldsymbol{\Sigma}\left(\gamma_{k} \boldsymbol{I}-\boldsymbol{D}^{-1 / 2} \hat{\boldsymbol{c}}_{k} \hat{\boldsymbol{c}}_{k}^{*} \boldsymbol{D}^{-1 / 2}\right) \boldsymbol{\Sigma}^{2} \boldsymbol{D}^{-1 / 2} \hat{\boldsymbol{c}}_{k}}
$$

with $\gamma_{k}=\left\|\boldsymbol{D}^{-1 / 2} \hat{\boldsymbol{c}}_{k}\right\|^{2}$. Reshuffling (??), the first-order TPE receiver admits the form

$\boldsymbol{w}_{k}^{\mathrm{TPE}} \propto \boldsymbol{D}^{-1} \hat{\boldsymbol{c}}_{k}-\frac{\psi_{k}}{1-\psi_{k}\left(\hat{\boldsymbol{c}}_{k}^{*} \boldsymbol{D}^{-1} \hat{\boldsymbol{c}}_{k}+\frac{\sigma^{2}}{P}\right)} \sum_{\ell \neq k} \hat{\boldsymbol{c}}_{\ell}^{*} \boldsymbol{D}^{-1} \hat{\boldsymbol{c}}_{k} \boldsymbol{D}^{-1} \hat{\boldsymbol{c}}_{\ell}$

which, recalling (??), can be interpreted as a linear PIC receiver. However, the TPE structure does not offer complete flexibility to set the weights regulating the interference cancellation: to match (??), it would be necessary that $\psi_{k}$ be a function of $\ell$, which the polynomial expansion does not allow.

\section{Performance Evaluation}

The performance is evaluated by means of the local-average SINR, $\mathbb{E}\left[\operatorname{sinr}_{k}\right]$ expected over the small-scale fading; this is an operationally significant quantity for the ergodic settings that are representative of coded communication in the face of diversity (in time, frequency, and/or space) and hybrid ARQ [?]. Shown in Fig. ?? is the distribution over the AP and user locations of the local-average SINR with $K / N=0.25$ and a single-slope pathloss. Besides MF, PIC, and MMSE, the figure includes the curve for PIC without the user-specific weights derived in (??) and the curve for first-order TPE. While, with the weights under which a TPE admits a PIC interpretation, and even without weighting, PIC uniformly improves upon the MF performance, the weights derived in the previous section are instrumental in realizing the potential of linear PIC reception. Henceforth, we consider only PIC receivers featuring such weights.

A similar improvement is observed for other loads $K / N$, with PIC doing slightly better under light loads than under heavy loads. Also, a commensurate improvement is observed (see Fig. ??) under a dual-slope pathloss model with breakpoint distance equal to $d$ as defined in Section ??. And, as Fig. ?? evidences, the behavior is robust over a wide range of dual-slope breakpoint distances. Interestingly, in terms of average performance there is an optimum network densityslightly different depending on the type of receiver-relative to the breakpoint distance: from the vantage of user $k$, higher network densities cause too many other users to be within the breakpoint distance of the APs capturing most power of user $k$, hence strongly interfering with it; lower densities cause user $k$ to be outside the breakpoint distance of most APs, reducing its received power.

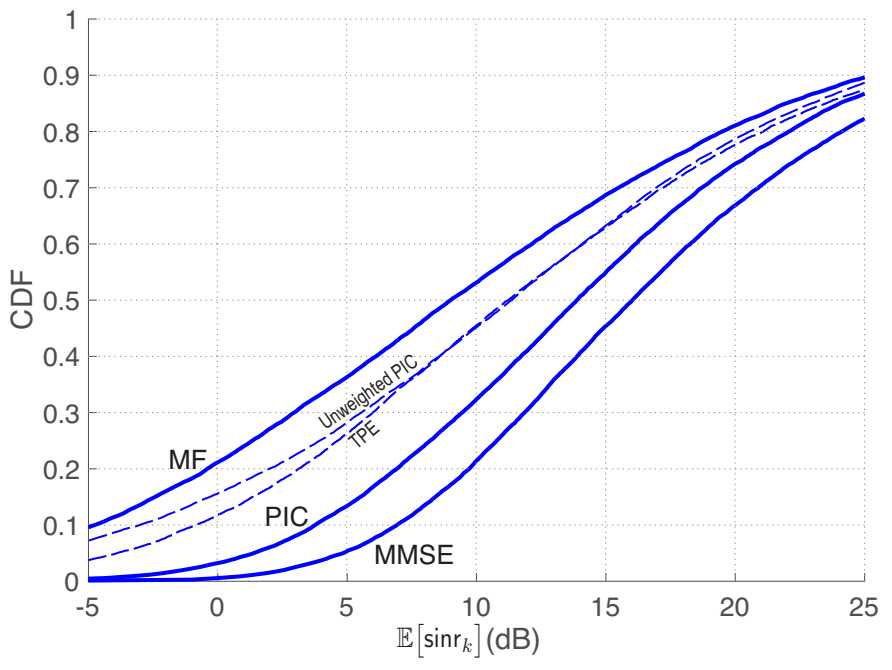

Fig. 3. CDF of the local-average user SINR for MF, linear PIC, and MMSE receivers, with $K / N=0.25$ and $\eta=4$. Also shown are the curves for unweighted PIC and first-order TPE.

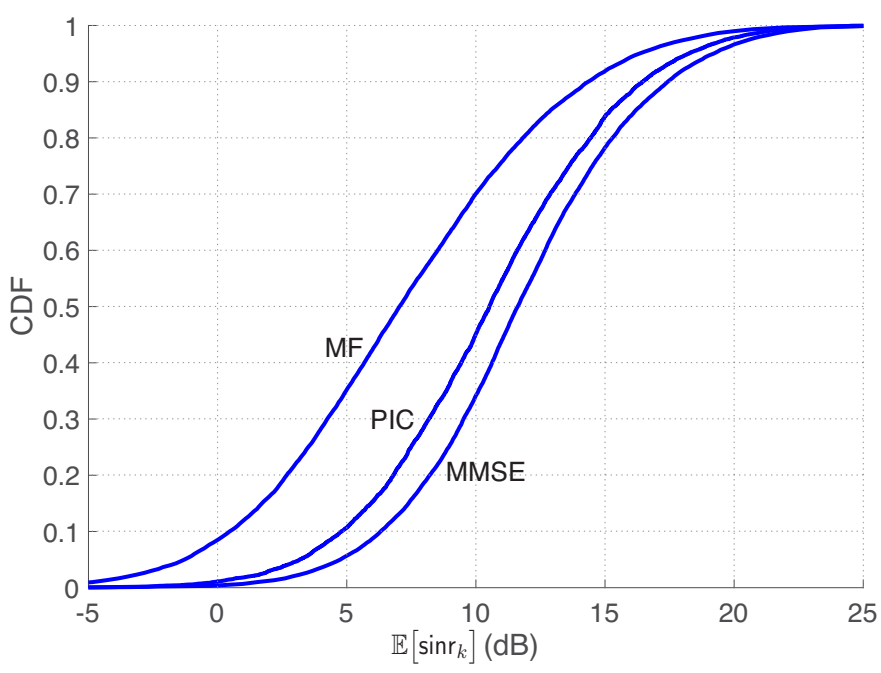

Fig. 4. CDF of the local-average user SINR for MF, linear PIC, and MMSE receivers, with $K / N=0.25$ and a dual-slope pathloss with breakpoint distance equal to $d$.

\section{Scalability}

A network-wide receiver encompassing the entire C-RAN is not scalable and, in large deployments with thousands of APs and users, it is outright unfeasible. Moreover, a network-wide receiver is an unnecessary overkill because, due to pathloss and shadowing, only a small share of APs capture substantial power from user $k$ and only a small share of other users cause substantial interference to user $k$. This suggests that somein fact the vast majority of - channel coefficients should be disregarded in terms of both signal reception and interference cancellation, and the estimation of those channel coefficients should be foregone altogether.

Let us consider the scalability of the receiver in terms of those aspects that are inherent to a C-RAN, namely (i) receiver computational cost, and (ii) channel estimation. The decoding and remaining post-processing tasks are as in a cellular network, one chain per user, hence inherently scalable. 


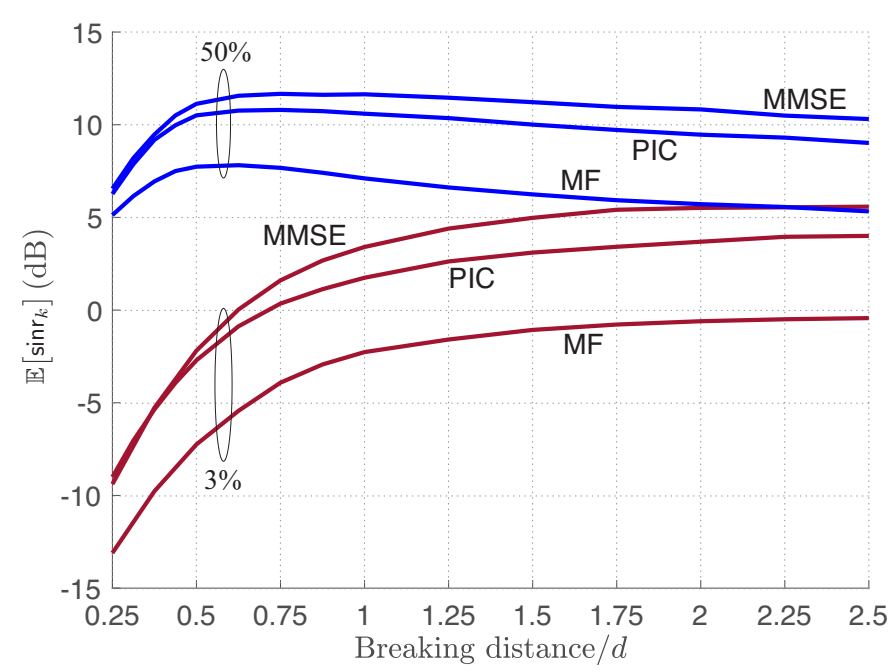

Fig. 5. $3 \%$ and $50 \%$ local-average user SINR levels as a function of the pathloss breakpoint distance normalized by $d$, with $K / N=0.25$.

We measure the cost, denoted by $M$, by the number of complex multiply-and-accumulate (MA) operations accrued computing and applying the receiver coefficients. In turn, we denote by $L$ the number of channel coefficients to be estimated. For growing $N$ and $K$, with some fixed ratio $K / N$, we want $M / N=\mathcal{O}(1)$ and $L / N=\mathcal{O}(1)$ as in a cellular network.

Measured in MA operations, the cost of $N \times N$ matrix inversions or multiplications is $\mathcal{O}\left(N^{3}\right)$ while the multiplication of $N \times K$ and $K \times N$ matrices costs $\mathcal{O}\left(K N^{2}\right)$. Our goal here is not to present a detailed complexity analysis, which would require positing specific implementations, but rather to establish scalability. With this in mind, these measures suffice and simpler operations such as additions can be neglected, leading to the following considerations:

- The whitening MF is readily available from the channel estimates, and its application to the observation $y$ incurs a cost per AP of

$$
\frac{M^{\mathrm{MF}}}{N}=\mathcal{O}(K)
$$

- The MMSE cost is dominated by the computation of (??), which satisfies

$$
\frac{M^{\mathrm{MMSE}}}{N}=\mathcal{O}\left(K^{2}\right)
$$

The TPE cost is somewhat lower thanks to the absence of inversions, but its scaling is no better than (??).

- For PIC, provided $\operatorname{sinr}_{0}^{\mathrm{MF}}, \ldots \operatorname{sinr}_{K-1}^{\mathrm{MF}}$ are available or can be measured directly, $a_{0}, \ldots, a_{K-1}$ require computing $\hat{\boldsymbol{c}}_{\ell}^{*} \boldsymbol{D}^{-1} \hat{\boldsymbol{c}}_{\ell}$ for $\ell=0, \ldots, K-1$, with a cost of $\mathcal{O}(K N)$, and then producing $\hat{x}_{0}, \ldots, \hat{x}_{K-1}$ again incurs a cost of $\mathcal{O}(K N)$. Likewise, (??) and the subsequent MF application both have a cost of $\mathcal{O}(K N)$. All in all,

$$
\frac{M^{\mathrm{PIC}}}{N}=\mathcal{O}(K)
$$

- For all the receiver types, $L / N=\mathcal{O}(K)$.

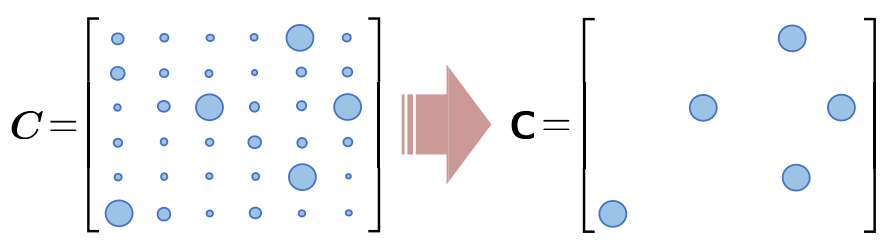

Fig. 6. Direct sparsification of the channel matrix.

Although the PIC receiver does involve additional operations relative to an $\mathrm{MF}$, the computational cost per AP is $\mathcal{O}(K)$ in both cases, as opposed to $\mathcal{O}\left(K^{2}\right)$ in the MMSE case. The PIC receiver thus goes a long way towards reconciling the MMSE performance with the MF cost. But the challenge remains to render the $\mathrm{PIC}$ receiver truly scalable, meaning $M^{\mathrm{PIC}} / N=\mathcal{O}(1)$ and $L^{\mathrm{PIC}} / N=\mathcal{O}(1)$, without compromising its performance.

\section{SPARSE PIC}

Even though, because of pathloss and shadowing, the channel matrix $\boldsymbol{C}$ has most of its mass concentrated on a small share of its entries, network-wide receivers estimate and process every entry of $\boldsymbol{C}$. This overreach afflicts the networkwide MF, MMSE, and PIC receivers alike. In all cases, the path to scalability lies precisely in recognizing and exploiting the nature of $\boldsymbol{C}$.

An intuitive idea could be to zero out all but the dominant entries of $\boldsymbol{C}$, as in Fig. ??, thereby obtaining a sparse matrix C to be plugged into the various expressions in lieu of $C$ itself. Unfortunately, such a direct sparsification might yield a channel matrix that is sparse, but unbalanced, with some users heavily favored by many connections while others are outright disconnected from the network. Likewise, some APs might be essentially taken out of service. The goal is therefore to generate a sparse channel matrix that is balanced across rows and columns, and to then capitalize on this sparsity to obtain a receiver structure that is scalable. This objective is tackled in [?] for MMSE reception, with the key to the derived solution being the delineation of a suitable subset of users to be processed by each AP and a suitable subset of APs to serve each user. We next tackle the same challenge in the context of PIC reception.

\section{A. Formulation}

Let us restrict to a subset, denoted by $\mathcal{K}_{n}$, the users whose channels are estimated by the $n$th AP; users not in $\mathcal{K}_{n}$ are treated as noise by such AP. Then, (??) can be rewritten as

$$
y_{n}=\sum_{k \in \mathcal{K}_{n}} \hat{c}_{n, k} x_{k}+\underbrace{\sum_{k \notin \mathcal{K}_{n}} \hat{c}_{n, k} x_{k}+z_{n}}_{\mathbf{z}_{n}}
$$

from which (??) changes to

$$
\boldsymbol{y}=\left[\begin{array}{c}
y_{0} \\
\vdots \\
y_{N-1}
\end{array}\right]=\hat{\mathbf{C}} \boldsymbol{x}+\mathbf{z}
$$


with $\mathbf{z}=\left[\mathrm{z}_{0} \cdots \mathrm{z}_{N-1}\right]^{\mathrm{T}}$,

$$
[\hat{\mathbf{C}}]_{n, k}=\left\{\begin{array}{cl}
{[\hat{\boldsymbol{C}}]_{n, k}} & k \in \mathcal{K}_{n} \\
0 & \text { otherwise }
\end{array}\right.
$$

and $\mathbb{E}\left[\mathbf{z z}^{*}\right]=\sigma^{2} \mathbf{D}$ where

$$
\begin{aligned}
{[\mathbf{D}]_{n, n} } & =\sum_{k \notin \mathcal{K}_{n}} \frac{\mathrm{SNR}_{n, k}^{2}}{1+\mathrm{SNR}_{n, k}}+[\boldsymbol{D}]_{n, n} \\
& =1+\sum_{k \in \mathcal{K}_{n}} \frac{\mathrm{SNR}_{n, k}}{1+\mathrm{SNR}_{n, k}}+\sum_{k \notin \mathcal{K}_{n}} \mathrm{SNR}_{n, k} .
\end{aligned}
$$

The signals being borne by the zeroed-out entries of $\hat{\mathbf{C}}$ have been moved to the augmented noise $\mathbf{z}$ and, with a proper choice of $\mathcal{K}_{0}, \ldots, \mathcal{K}_{N-1}$, the rows of $\hat{\mathbf{C}}$ have a balanced number of nonzero entries.

Next, let us curb to a subset $\mathcal{N}_{k}$ the APs that participate in the reception of user $k$. This can be modelled as a decimation of $\hat{\mathbf{C}}$, which is the approach taken in [?], but also as a further pruning, which is more convenient to the present formulation. Precisely, we conduct a zeroing process dual to that of (??), namely

$$
[\hat{\mathbf{C}}]_{n, k}=\left\{\begin{array}{cl}
{[\hat{\mathbf{C}}]_{n, k}} & n \in \mathcal{N}_{k} \\
0 & \text { otherwise },
\end{array}\right.
$$

and, with a proper choice of $\mathcal{N}_{k}$, the columns of the final $\hat{\mathbf{C}}$ are also balanced for every $k$. Note how, for $\left|\mathcal{K}_{n}\right|=K$ and $\left|\mathcal{N}_{k}\right|=N$, we have $\hat{\mathbf{C}}=\hat{\boldsymbol{C}}$ such that we recover the original channel matrix.

Based on the sparsified channel, the PIC for user $k$ is applied as

$$
\boldsymbol{y}-\sum_{\ell \neq k} \hat{\mathbf{c}}_{\ell} \hat{x}_{\ell}
$$

where

$$
\hat{x}_{\ell}=\mathrm{a}_{\ell} \hat{\mathbf{c}}_{\ell}^{*} \mathbf{D}^{-1} \boldsymbol{y}
$$

and

$$
\mathrm{a}_{\ell}=\frac{\sin r_{\ell}^{\mathrm{MF}}}{1+\operatorname{sinr}_{\ell}^{\mathrm{MF}}} \frac{1}{\hat{\mathbf{c}}_{\ell}^{*} \mathbf{D}^{-1} \hat{\mathbf{c}}_{\ell}}
$$

with $\operatorname{sinr}_{\ell}^{\mathrm{MF}}$ the value obtained when user $\ell$ is MF-received by the APs that constitute $\mathcal{N}_{\ell}$. Altogether, the linear PIC receiver for user $k$ is now

$$
\mathbf{w}_{k}^{\mathrm{PIC}}=\mathbf{D}^{-1} \hat{\mathbf{c}}_{k}-\sum_{\ell \neq k} \frac{\sin r_{\ell}^{\mathrm{MF}}}{1+\operatorname{sinr}_{\ell}^{\mathrm{MF}}} \frac{\hat{\mathbf{c}}_{\ell}^{*} \mathbf{D}^{-1} \hat{\mathbf{c}}_{\ell}}{\hat{\mathbf{c}}_{\ell}^{*} \mathbf{D}^{-1} \hat{\mathbf{c}}_{\ell}} \mathbf{D}^{-1} \hat{\mathbf{c}}_{\ell}
$$

and

$$
\operatorname{sinr}_{k}^{\mathrm{PIC}}=\frac{\left|\mathbf{w}_{k}^{\mathrm{PIC} *} \hat{\mathbf{c}}_{k}\right|^{2}}{\sum_{\ell \neq k}\left|\mathbf{w}_{k}^{\mathrm{PIC} *} \hat{\mathbf{c}}_{\ell}\right|^{2}+\frac{\sigma^{2}}{P} \mathbf{w}_{k}^{\mathrm{PIC} *} \mathbf{D} \mathbf{w}_{k}^{\mathrm{PLC}}} .
$$

What remains is to see how to determine the composition of the subsets $\mathcal{N}_{k}$ and $\mathcal{K}_{n}$ to ensure that $\hat{\mathbf{C}}$ is sparse, but balanced, and that the performance of the network-wide PIC receiver is approached as closely as desired. These subsets should be determined from large-scale quantities only, such that they are relatively stable in time and frequency and do not need to be redefined at the fading scales of milliseconds and hundreds of kilohertz.

\section{B. AP Subset Selection}

For user $k$, the number of potential AP subsets of size $\left|\mathcal{N}_{k}\right|$ equals

$$
\frac{N !}{\left(\left|\mathcal{N}_{k}\right|\right) !\left(N-\left|\mathcal{N}_{k}\right|\right) !},
$$

hence an exhaustive inspection is out of the question. Rather, to select $\mathcal{N}_{0}, \ldots, \mathcal{N}_{K-1}$ we embrace the policy propounded in [?], [?], [?] for MF and in [?], [?] for MMSE reception, whereby $\mathcal{N}_{k}$ contains the $\left|\mathcal{N}_{k}\right|$ APs whose $G_{n, k}$ is largest, i.e., to which user $k$ has the strongest links.

\section{User Subset Selection}

In terms of the selection of $\mathcal{K}_{n}$, a dual policy of the one adopted for $\mathcal{N}_{k}$ seems like a sensible solution. However, an added requirement exists: the $n$th AP should always estimate the channels of users in whose reception it participates. We hence propose that $\mathcal{K}_{n}$ contain the union of:

- A fixed number of the users whose $G_{n, k}$ are largest, i.e., with strongest links to AP $n$.

- All users for which $n \in \mathcal{N}_{k}$, i.e., all users being received by AP $n$.

\section{Scalability}

Computing $\mathrm{a}_{0}, \ldots, \mathrm{a}_{K-1}$ requires $\hat{\mathbf{c}}_{\ell}^{*} \mathbf{D}^{-1} \hat{\mathbf{c}}_{\ell}$ for $\ell=$ $0, \ldots, K-1$, with a cost of $\mathcal{O}\left(\sum_{\ell=0}^{K-1}\left|\mathcal{N}_{k}\right|\right)$, and subsequently producing $\hat{x}_{0}, \ldots, \hat{x}_{K-1}$ has a cost of the same order. Likewise, (??) and the necessary MF applications have a cost of that same order. All in all, the cost of obtaining and applying $\mathbf{w}_{0}^{\mathrm{PIC}}, \ldots, \mathbf{w}_{K-1}^{\mathrm{PIC}}$ satisfies

$$
\frac{M^{\mathrm{PIC}}}{N}=\mathcal{O}\left(\frac{\sum_{k=0}^{K-1}\left|\mathcal{N}_{k}\right|}{N}\right),
$$

which is $\mathcal{O}(1)$ for fixed $K / N$. In turn, the number of channel estimates satisfies

$$
\frac{L^{\mathrm{PlC}}}{N}=\frac{\sum_{n=0}^{N-1}\left|\mathcal{K}_{n}\right|}{N}
$$

which is again $\mathcal{O}(1)$.

To provide some perspective, (??) and (??) can be contrasted with their counterparts for MMSE reception, subject to the same AP and user subsets [?]. As it is, the number of channel estimates abides exactly by (??) while the cost of obtaining and applying $\mathbf{w}_{0}^{\text {MMSE }}, \ldots, \mathbf{w}_{K-1}^{\text {MMSE }}$ satisfies

$$
\frac{M^{\mathrm{MMSE}}}{N}=\mathcal{O}\left(\frac{\sum_{k=0}^{K-1}\left|\mathcal{N}_{k}\right|^{3}}{N}\right),
$$

also $\mathcal{O}(1)$ but substantially higher than for PIC. Hence, a sparse PIC receiver retains a computational advantage with respect to the MMSE benchmark. 


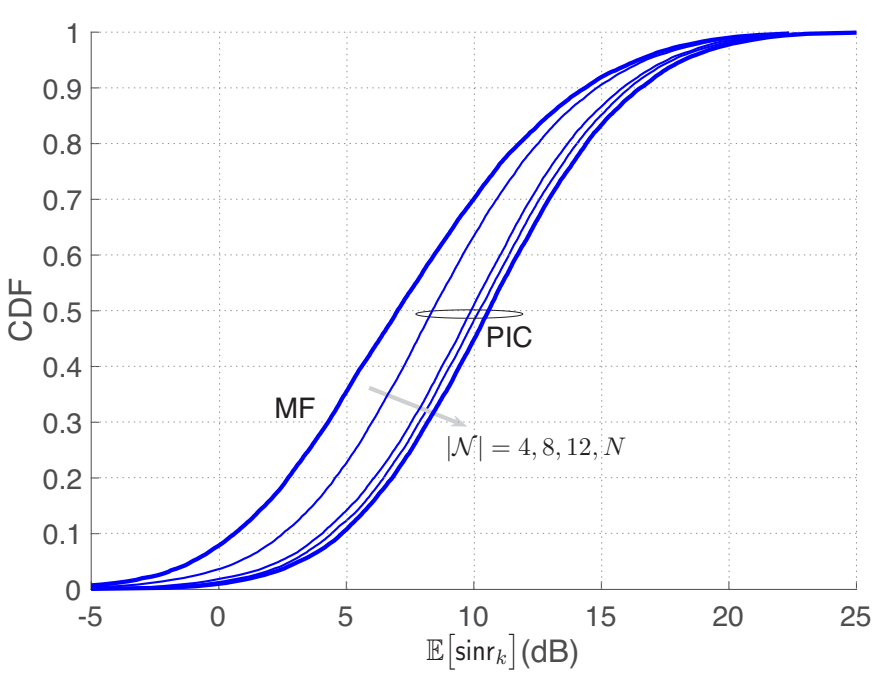

Fig. 7. CDF of the local-average user SINR for MF and linear PIC receivers with $K / N=0.25$ and a dual-slope pathloss, for a breakpoint distance equal to $d$. The PIC performance is parameterized by $|\mathcal{N}|$, with $|\mathcal{K}| /|\mathcal{N}|=K / N$.

\section{E. Performance Evaluation}

Let the AP subset size $|\mathcal{N}|$ be identical for all users, while the user subsets $\mathcal{K}_{0}, \ldots, \mathcal{K}_{N-1}$ contain the $\frac{K}{N}|\mathcal{N}|$ users with strongest channels to the respective APs in union with the users being received by each such AP. The cardinality of the user subsets therefore varies across APs. Presented in Fig. ?? is the distribution of the user SINR with sparse PIC reception, parameterized by $|\mathcal{N}|$. Remarkably, very small subset sizes suffice to essentially match the performance of network-wide PIC reception. Specifically, it suffices to have every user be received by about 8 APs with the 2-3 strongest interferers cancelled. This second aspect, namely that a few terms dominate the interference suffered by a given user, is in agreement with observations made, in the context of MMSE reception, in works such as [?], [?], [?].

\section{POWER CONTROL}

Because of the near-far effect, whereby users close to an AP may overwhelm the weaker signals from more distant users, power control is a very important feature in the uplink of wireless networks in general. This is also the case in cell-free networks, where the role of the cellular AP is played by the entire set of APs participating in the reception: if $\left\|c_{k}\right\|^{2}$ is large while if $\left\|\boldsymbol{c}_{\ell}\right\|^{2}$ is small, then the interference from user $k$ is likely to engulf the signal of user $\ell$.

Power control is particularly important to ensure acceptable large-scale outage levels, meaning the share of users whose local-average SINR is below some acceptable threshold. In this respect, the worst-case pathloss model is the single-slope one, hence we abide by that model in this section. We also revert back to network-wide reception, but any power control solution is readily applicable with sparse reception as well. Establishing $-5 \mathrm{~dB}$ as a reasonable threshold, we observe from Fig. ?? that, while the MF receiver exhibits a fairly high outage level, the PIC receiver is essentially free of outages. This indicates that, with fixed-power transmissions, an MF receiver necessitates of a lower load $K / N$, as that is the mechanism available to correct the outage performance in the absence of power control, while a PIC receiver can afford a higher load. The introduction of power control has the benefit of providing an additional and powerful mechanism to improve the outage performance.

With power control coefficients incorporated, the vector of AP observations becomes

$$
\boldsymbol{y}=\sqrt{P} \sum_{k=0}^{K-1} \hat{\boldsymbol{c}}_{k} \sqrt{p_{k}} s_{k}+\boldsymbol{z},
$$

where $s_{k}$ is the unit-power symbol transmitted by user $k$ and $p_{k} \in[0,1]$ is its power control coefficient. The linear PIC receiver for user $k$ continues to be given by

$$
\boldsymbol{w}_{k}^{\mathrm{PIC}}=\boldsymbol{D}^{-1} \hat{\boldsymbol{c}}_{k}-\sum_{\ell \neq k} \frac{\operatorname{sinr}_{\ell}^{\mathrm{MF}}}{1+\operatorname{sinr}_{\ell}^{\mathrm{MF}}} \frac{\hat{\boldsymbol{c}}_{\ell}^{*} \boldsymbol{D}^{-1} \hat{\boldsymbol{c}}_{k}}{\hat{\boldsymbol{c}}_{\ell}^{*} \boldsymbol{D}^{-1} \boldsymbol{c}_{\ell}} \boldsymbol{D}^{-1} \hat{\boldsymbol{c}}_{\ell}
$$

only with $\operatorname{sinr}_{\ell}^{\mathrm{MF}}$ generalized to

$$
\operatorname{sinr}_{\ell}^{\mathrm{MF}}=\frac{p_{\ell}\left|\hat{\boldsymbol{c}}_{\ell}^{*} \boldsymbol{D}^{-1} \hat{\boldsymbol{c}}_{\ell}\right|^{2}}{\sum_{j \neq \ell} p_{j}\left|\hat{\boldsymbol{c}}_{\ell}^{*} \boldsymbol{D}^{-1} \hat{\boldsymbol{c}}_{j}\right|^{2}+\frac{\sigma^{2}}{P} \hat{\boldsymbol{c}}_{\ell}^{*} \boldsymbol{D}^{-1} \hat{\boldsymbol{c}}_{\ell}},
$$

and, at the output of $\boldsymbol{w}_{k}^{\mathrm{PIC}}$, we attain

$$
\operatorname{sinr}_{k}^{\mathrm{PIC}}=\frac{p_{k}\left|\boldsymbol{w}_{k}^{\mathrm{PIC} *} \hat{\boldsymbol{c}}_{k}\right|^{2}}{\sum_{\ell \neq k} p_{\ell}\left|\boldsymbol{w}_{k}^{\mathrm{PIC} *} \hat{\boldsymbol{c}}_{\ell}\right|^{2}+\frac{\sigma^{2}}{P} \boldsymbol{w}_{k}^{\mathrm{PIC} *} \boldsymbol{D} \boldsymbol{w}_{k}^{\mathrm{PIC}}},
$$

which generalizes (??).

The problem is now to adjust $p_{0}, \ldots, p_{K-1}$ in order to optimize some desired loss function of $\operatorname{sinr}_{0}^{\mathrm{PIC}}, \ldots, \operatorname{sinr}_{K-1}^{\mathrm{PIC}}$. A key desideratum for an uplink power control policy is that $p_{k}$ depend only on $\left\{G_{n, k}\right\}$, but not on rapidly varying small-scale quantities. This requires expressing $\mathbb{E}\left[\sin r_{k}\right]$, or some approximation thereof, explicitly as a function of only $p_{0}, \ldots, p_{K-1}$ and $\left\{G_{n, k}\right\}$. For MF reception, such approximations exist [?, eq. 27], setting the stage for power control schemes based on:

- Convex optimization. This approach is restricted to SINR loss functions that can be cast as convex or quasi-convex in $p_{0}, \ldots, p_{K-1}$, and it does not scale well to large dimensionalities, but it does cover the arguably most popular loss function, the max-min loss [?]. This loss function is very effective at minimizing outages, even if at a steep price in the overall performance, which is dragged down by worst-case users [?].

- Learning schemes operating on neural networks. This approach scales somewhat better and, in principle, it is universal in the SINR loss functions, even if without guarantees of global optimality. Existing results do indicate a solid performance for various loss functions of interest [?], [?].

Adapting the above schemes to PIC reception would require, as a first step, expressing $\mathbb{E}\left[\operatorname{sinr}_{k}^{\mathrm{PIC}}\right]$ explicitly as a function of only $p_{0}, \ldots, p_{K-1}$ and $\left\{G_{n, k}\right\}$, with all the small-scale quantities expected over. As this appears to be an unwieldy task, the research challenge here would be to find an approximation or proxy for $\mathbb{E}\left[\operatorname{sinr}_{k}^{\mathrm{PIC}}\right]$ that meets this condition. To apply convex tools, moreover, this should then lead to a convex SINR loss 
function of the powers. We do not dwell on this challenge here, but rather take a different route. Precisely, in the reminder we concentrate on fractional power control, which has long been the reigning alternative for the uplink of cellular networks.

The seed for fractional power control can be found in [?], which derived the transmit powers minimizing the variance of the SIR distribution (in $\mathrm{dB}$ ) experienced by two interfering cellular users; the found solution was $p_{k} \propto 1 / \sqrt{G_{k}}$ with $G_{k}$ the large-scale gain to the serving cell and with the proportionality factor ensuring that $p_{k} \in[0,1]$. This solution was subsequently generalized to [?], [?]

$$
p_{k} \propto \frac{1}{G_{k}^{\vartheta}}
$$

where the parameter $\vartheta \in[0,1]$ regulates the extent to which CDFs such as the ones in Fig. ?? become steeper: higher values reduce the outages further, at the expense of a lower average performance.

Fractional power control is simple, yet surprisingly effective because it captures the essence of the near-far effect in a cellular network and it can compensate for it effectively: users power down as their large-scale gain to the serving AP increases, and vice versa, with the single parameter $\vartheta$ controlling the intensity of this correction. In the cell-free case, all APs are intended, hence the role of the large-scale gain for user $k$ would seem to be played by $\sum_{n=0}^{N-1} G_{n, k}$. Indeed, fractional power control generalizes to cell-free networks with MF reception as [?]

$$
p_{k} \propto \frac{1}{\left(\sum_{n=0}^{N-1} G_{n, k}\right)^{\vartheta}}
$$

and its performance continues to be excellent.

Albeit derived under MF reception, (??) turns out to work well with PIC reception too-with $\vartheta$ shifted to smaller values so as to allow for higher transmit powers given the receiver's ability to suppress some of the interference. To test (??) under stress, we aggressively push the load $K / N$ close to unity and establish $3 \%$ as the acceptable outage level at $-5 \mathrm{~dB}$ of SINR. Illustrated in Fig. ?? is the performance for $K / N=0.8$ of MF and PIC reception with fractional power control adjusted such that the $3 \%$ outage level is $-5 \mathrm{~dB}$ : this corresponds to $\vartheta=1$ for MF and $\vartheta=0.65$ for PIC.

Power control does not diminish the benefits of PIC reception relative to $\mathrm{MF}$, it merely displaces those benefits from the lower to the upper part of the CDF. With the outage level anchored, it is now the average and the peak performance levels that display marked improvements. And it may well be possible to tweak the fractional power control policy in order to better capitalize on the interference rejection mechanism of PIC receivers, further improving their perfomance advantage. This is another avenue for follow-up research.

\section{CONCLUSION}

A one-shot linear receiver based on a single stage of PIC with user-specific weights is an attractive alternative to MF beamforming in terms of performance, and to MMSE

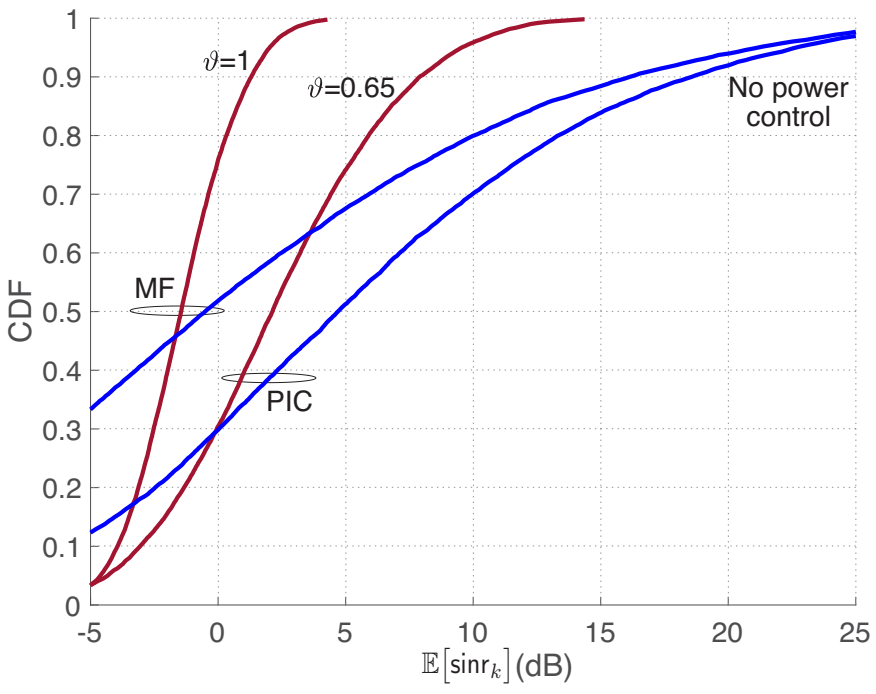

Fig. 8. CDF of the local-average user SINR for MF and linear PIC networkwide receivers for $K / N=0.8$ and $\eta=4$, without and with fractional power control. The parameter $\vartheta$ is set such that the $3 \%$ outage level is $-5 \mathrm{~dB}$.

filtering in terms of computational cost, for C-RAN reception. Reinforcing these arguments, a PIC structure is inherently amenable to parallelization, which is often as relevant as the raw computational cost itself. The crux of the linear PIC receiver is the tilting of each user's beam away from the interference of other users, conservatively with respect to an MMSE receiver (which orients the beams optimally in terms of the SINRs), but at a much reduced cost and notably without the need for matrix inversions.

A natural question is whether, with additional PIC stages, the orientation of the beams would edge further towards optimality, and the answer is affirmative. An illuminating result in this respect is presented in Fig. ??, which depicts the performance (for network-wide reception without power control) as a function of the SNR at a distance $d$. The MMSE receiver, eventually behaving as a zero-forcer, improves steadily with the SNR. In contrast, the MF, being oblivious to the interference, exhibits a curbed SINR and is unable to take advantage of higher transmit powers or increased network densities. The performance of the PIC receiver is curbed higher above and, with a second stage (see Appendix ?? for the formulation), it bends towards the MMSE benchmark. Additional stages would bend it progressively because, as shown in [?], each soft PIC stage can be interpreted as a step in the gradient descend towards the MMSE solution. At the same time, since every stage incurs a cost per $\mathrm{AP}$ of $M^{\mathrm{PIC}} / N=\mathcal{O}(K)$, by the time the number of stages reached $K$, the cost per AP would be $\mathcal{O}\left(K^{2}\right)$ as in the MMSE receiver. What makes the linear PIC architecture interesting is that, with the weighting derived in this paper, most performance benefits are reaped with a single stage, hence with the most savings in cost.

Then, with a suitable sparsification of the overall channel matrix, the PIC receiver can be rendered scalable in terms of both cost and channel estimation (which directly maps to pilot overhead). This sparsification entails a careful definition of the subset of APs that receive each user, and of the subset 


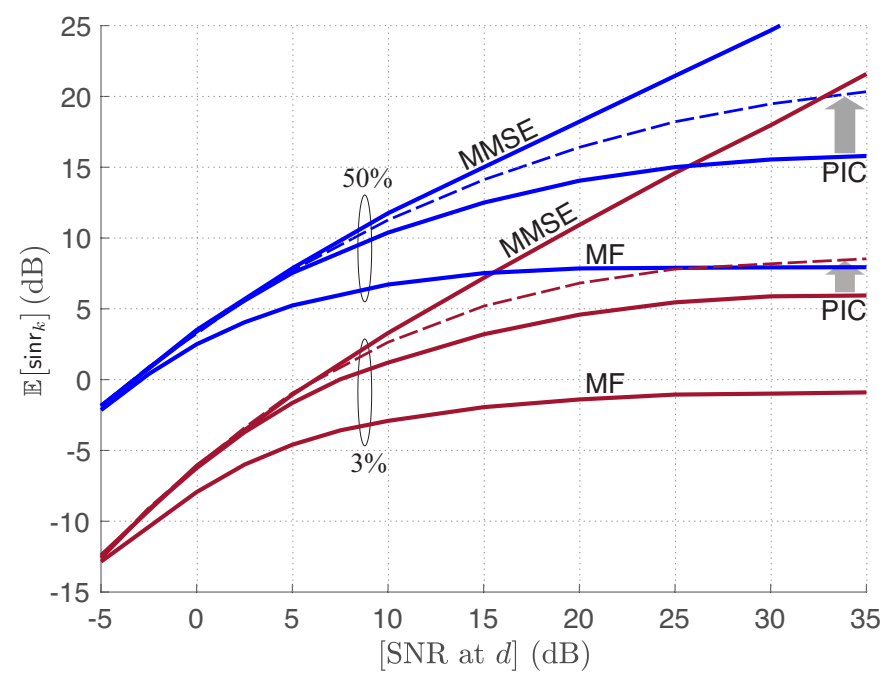

Fig. 9. $3 \%$ and $50 \%$ local-average user SINRs as a function of the SNR at distance $d$ with network-wide reception, $K / N=0.25$, and a two-slope pathloss model. In solid: MF, MMSE, and PIC receivers. In dashed: two-stage PIC receiver.

of users received by each AP, striking a balance between capturing the strongest links in the network and ensuring that no users are starved of service nor are any APs disconnected. In our results, the cardinalities of these subsets have been kept fixed, which leaves room for additional improvements if they were instead separately optimized; this constitutes yet another potential subject of future research.

Pilot contamination has been disregarded in our performance evaluations, and it would certainly be of interest to refine the results with it. That said, it must be born in mind that ultradense deployments exhibit (because of their short range) very high frequency coherences and, most likely, also very high time coherences. Altogether, the fading coherence is sure to be in the thousands of resource units, a very benevolent situation as far as pilot contamination is concerned. For instance, for very conservative coherences of $B_{\mathrm{c}}=2$ $\mathrm{MHz}$ in frequency and $T_{\mathrm{c}}=5 \mathrm{~ms}$ in time, we would have roughly $B_{\mathrm{c}} T_{\mathrm{c}}=10^{4}$ resource units of fading coherence and, allocating only $10 \%$ of those to pilots, we would have approximately 1000 pilot dimensions. This is already an order of magnitude more than the number of pilots required for contamination to become unimportant under MF reception and random pilot assignment [?], let alone with contamination mitigation procedures such as the ones propounded in [?, sec. IV] or in [?], [?], [?], [?].

\section{ACKNOWLEDGMENT}

The handling by the Associate Editor, and the feedback provided by the anonymous reviewers, are gratefully acknowledged.

\section{APPENDIX A}

VERIFICATION OF (??)

Plugging (??) into (??),

$\boldsymbol{w}_{k}^{\mathrm{PIC}}=\boldsymbol{D}^{-1} \hat{\boldsymbol{c}}_{k}-\sum_{\ell \neq k} \hat{\boldsymbol{c}}_{\ell}^{*} \boldsymbol{D}^{-1} \hat{\boldsymbol{c}}_{k}\left(\hat{\boldsymbol{C}} \hat{\boldsymbol{C}}^{*}+\frac{\sigma^{2}}{P} \boldsymbol{D}\right)^{-1} \hat{\boldsymbol{c}}_{\ell}$

$$
\begin{aligned}
= & \boldsymbol{D}^{-1} \hat{\boldsymbol{c}}_{k}-\sum_{\ell \neq k}\left(\hat{\boldsymbol{C}} \hat{\boldsymbol{C}}^{*}+\frac{\sigma^{2}}{P} \boldsymbol{D}\right)^{-1} \hat{\boldsymbol{c}}_{\ell} \hat{\boldsymbol{c}}_{\ell}^{*} \boldsymbol{D}^{-1} \hat{\boldsymbol{c}}_{k} \\
= & \left(\hat{\boldsymbol{C}} \hat{\boldsymbol{C}}^{*}+\frac{\sigma^{2}}{P} \boldsymbol{D}\right)^{-1}\left(\hat{\boldsymbol{C}} \hat{\boldsymbol{C}}^{*}+\frac{\sigma^{2}}{P} \boldsymbol{D}\right) \boldsymbol{D}^{-1} \hat{\boldsymbol{c}}_{k} \\
& -\sum_{\ell \neq k}\left(\hat{\boldsymbol{C}} \hat{\boldsymbol{C}}^{*}+\frac{\sigma^{2}}{P} \boldsymbol{D}\right)^{-1} \hat{\boldsymbol{c}}_{\ell} \hat{\boldsymbol{c}}_{\ell}^{*} \boldsymbol{D}^{-1} \hat{\boldsymbol{c}}_{k} \\
= & \left(\hat{\boldsymbol{C}} \hat{\boldsymbol{C}}^{*}+\frac{\sigma^{2}}{P} \boldsymbol{D}\right)^{-1}\left[\left(\hat{\boldsymbol{C}} \hat{\boldsymbol{C}}^{*}+\frac{\sigma^{2}}{P} \boldsymbol{D}\right)-\sum_{\ell \neq k} \hat{\boldsymbol{c}}_{\ell} \hat{\boldsymbol{c}}_{\ell}^{*}\right] \boldsymbol{D}^{-1} \hat{\boldsymbol{c}}_{k} \\
= & \left(\hat{\boldsymbol{C}} \hat{\boldsymbol{C}}^{*}+\frac{\sigma^{2}}{P} \boldsymbol{D}\right)^{-1}\left(\hat{\boldsymbol{c}}_{k} \hat{\boldsymbol{c}}_{k}^{*}+\frac{\sigma^{2}}{P} \boldsymbol{D}\right) \boldsymbol{D}^{-1} \hat{\boldsymbol{c}}_{k} \\
= & \left(\hat{\boldsymbol{C}} \hat{\boldsymbol{C}}^{*}+\frac{\sigma^{2}}{P} \boldsymbol{D}\right)^{-1}\left(\hat{\boldsymbol{c}}_{k} \hat{\boldsymbol{c}}_{k}^{*} \boldsymbol{D}^{-1} \hat{\boldsymbol{c}}_{k}+\frac{\sigma^{2}}{P} \hat{\boldsymbol{c}}_{k}\right) \\
= & \left(\hat{\boldsymbol{C}} \hat{\boldsymbol{C}}^{*}+\frac{\sigma^{2}}{P} \boldsymbol{D}\right)^{-1} \hat{\boldsymbol{c}}_{k}\left(\hat{\boldsymbol{c}}_{k}^{*} \boldsymbol{D}^{-1} \hat{\boldsymbol{c}}_{k}+\frac{\sigma^{2}}{P}\right) \\
= & \left(\hat{\boldsymbol{c}}_{k}^{*} \boldsymbol{D}^{-1} \hat{\boldsymbol{c}}_{k}+\frac{\sigma^{2}}{P}\right) \boldsymbol{w}_{k}^{\mathrm{MMSE}},
\end{aligned}
$$

where $\left(\hat{\boldsymbol{c}}_{k}^{*} \boldsymbol{D}^{-1} \hat{\boldsymbol{c}}_{k}+\frac{\sigma^{2}}{P}\right)$ is a real-valued scalar.

\section{APPENDIX B}

TWO-STAGE PIC

Iterating the procedure described in Section ??, we obtain

$$
\boldsymbol{y}_{k}^{(2)}=\boldsymbol{y}-\sum_{\ell \neq k} \hat{\boldsymbol{c}}_{\ell} \hat{x}_{\ell}^{(2)},
$$

where

$$
\begin{aligned}
\hat{x}_{\ell}^{(2)} & =a_{\ell}^{(2)} \hat{\boldsymbol{c}}_{\ell}^{*} \boldsymbol{D}^{-1} \boldsymbol{y}_{k} \\
& =a_{\ell}^{(2)} \boldsymbol{w}_{\ell}^{\mathrm{PIC} *} \boldsymbol{y},
\end{aligned}
$$

is the second-stage soft estimate of $x_{\ell}$, with

$$
\begin{aligned}
a_{\ell}^{(2)} & =\frac{\mathbb{E}\left[\boldsymbol{w}_{\ell}^{\mathrm{PIC} *} \boldsymbol{y} \cdot x_{\ell}^{*} \mid \boldsymbol{w}_{0}^{\mathrm{PIC}}, \ldots, \boldsymbol{w}_{K-1}^{\mathrm{PIC}}\right]}{\mathbb{E}\left[\boldsymbol{w}_{\ell}^{\mathrm{PI} C} \boldsymbol{y} \cdot \boldsymbol{y}^{*} \boldsymbol{w}_{\ell}^{\mathrm{PC}} \mid \boldsymbol{w}_{0}^{\mathrm{PCC}}, \ldots, \boldsymbol{w}_{K-1}^{\mathrm{PC}}\right]} \\
& =\frac{\boldsymbol{w}_{\ell}^{\mathrm{PC} *} \hat{\boldsymbol{c}}_{\ell}}{\sum_{j=0}^{K-1}\left|\boldsymbol{w}_{\ell}^{\mathrm{PIC} *} \hat{\boldsymbol{c}}_{j}\right|^{2}+\frac{\sigma^{2}}{P} \boldsymbol{w}_{\ell}^{\mathrm{PlC} *} \boldsymbol{D} \boldsymbol{w}_{\ell}^{\mathrm{PC}}},
\end{aligned}
$$

which can be seen to equal

$$
a_{\ell}^{(2)}=\frac{\operatorname{sinr}_{\ell}^{\mathrm{PC}}}{1+\operatorname{sinr}_{\ell}^{\mathrm{PlC}}} \frac{1}{\boldsymbol{c}_{\ell}^{*} \boldsymbol{w}_{\ell}} .
$$

Hence, the two-stage PIC linear receiver is given by

$$
\boldsymbol{w}_{k}^{\mathrm{PIC}(2)}=\boldsymbol{D}^{-1} \hat{\boldsymbol{c}}_{k}-\sum_{\ell \neq k} \frac{\operatorname{sinr}_{\ell}^{\mathrm{PC}}}{1+\operatorname{sinr}_{\ell}^{\mathrm{PIC}}} \frac{\hat{\boldsymbol{c}}_{\ell}^{*} \boldsymbol{D}^{-1} \hat{\boldsymbol{c}}_{k}}{\hat{\boldsymbol{c}}_{\ell}^{*} \boldsymbol{w}_{\ell}} \boldsymbol{w}_{\ell}
$$

and the SINR of user $k$ at its output equals

$$
\operatorname{sinr}_{k}^{\mathrm{PC}(2)}=\frac{\left|\boldsymbol{w}_{k}^{\mathrm{PLC}(2) *} \hat{\boldsymbol{c}}_{k}\right|^{2}}{\sum_{\ell \neq k}\left|\boldsymbol{w}_{k}^{\mathrm{PIC}(2) *} \hat{\boldsymbol{c}}_{\ell}\right|^{2}+\frac{\sigma^{2}}{P} \boldsymbol{w}_{k}^{\mathrm{PC}(2) *} \boldsymbol{D} \boldsymbol{w}_{k}^{\mathrm{PLC}(2)}} .
$$

\section{REFERENCES}

[1] R. Mosayebi, M. Mojahedian, and A. Lozano, "Parallel interference cancellation for cell-free C-RANs," in Asilomar Conf. on Signals, Systems and Computers, Nov. 2020.

[2] M. Dohler, R. W. Heath, A. Lozano, C. B. Papadias, and R. A. Valenzuela, "Is the PHY layer dead?" IEEE Communications Magazine, vol. 49, no. 4, pp. 159-165, Apr. 2011. 
[3] R. Irmer, J. Droste, P. Marsch, M. Grieger, G. Fettweis, S. Brueck, H.-P. Mayer, L. Thiele, and V. Jungnickel, "Coordinated multipoint: Concepts, performance, and field trial results," IEEE Commun. Magazine, vol. 49, no. 2, pp. 102-111, 2011.

[4] M. Sawahashi, Y. Kishiyama, A. Morimoto, D. Nishikawa, and M. Tanno, "Coordinated multipoint transmission/reception techniques for LTE-advanced," IEEE Wireless Commun., vol. 17, no. 3, p. 26, 2010.

[5] D. Lee, H. Seo, B. Clerckx, E. Hardouin, D. Mazzarese, S. Nagata, and K. Sayana, "Coordinated multipoint transmission and reception in LTE-advanced: deployment scenarios and operational challenges," IEEE Commun. Magazine, vol. 50, no. 2, pp. 148-155, 2012.

[6] X. Zhang and J. G. Andrews, "Downlink cellular network analysis with multi-slope path loss models," IEEE Trans. Commun., vol. 63, no. 5, pp. 1881-1894, May 2015.

[7] A. Checko, H. L. Christiansen, Y. Yan, L. Scolari, G. Kardaras, M. S. Berger, and L. Dittmann, "Cloud RAN for mobile networks-A technology overview," IEEE Commun. Surveys \& Tutorials, vol. 17, no. 1, pp. 405-426, 2014.

[8] P. Rost, C. J. Bernardos, A. De Domenico, M. Di Girolamo, M. Lalam, A. Maeder, D. Sabella, and D. Wübben, "Cloud technologies for flexible 5G radio access networks," IEEE Commun. Magazine, vol. 52, no. 5, pp. 68-76, 2014.

[9] S. Bhaumik, S. P. Chandrabose, M. K. Jataprolu, G. Kumar, A. Muralidhar, P. Polakos, V. Srinivasan, and T. Woo, "CloudIQ: A framework for processing base stations in a data center," in Int'l Conf. Mobile Computing and Netw., 2012, pp. 125-136.

[10] H. ElSawy, H. Dahrouj, T. Y. Al-Naffouri, and M.-S. Alouini, "Virtualized cognitive network architecture for 5G cellular networks," IEEE Commun. Magazine, vol. 53, no. 7, pp. 78-85, 2015.

[11] C. I, J. Huang, R. Duan, C. Cui, J. Jiang, and L. Li, "Recent progress on C-RAN centralization and cloudification," IEEE Access, vol. 2, pp. 1030-1039, 2014

[12] N. Lee, R. W. Heath, D. Morales-Jimenez, and A. Lozano, "Base station cooperation with dynamic clustering in super-dense cloud-RAN," in IEEE Globecom Workshops (GC'13 Wkshps), Dec. 2013, pp. 784-788.

[13] J. Wu, Z. Zhang, Y. Hong, and Y. Wen, "Cloud radio access network (C-RAN): a primer," IEEE Network, vol. 29, no. 1, pp. 35-41, Jan. 2015.

[14] P. Rost, S. Talarico, and M. C. Valenti, "The complexity-rate tradeoff of centralized radio access networks," IEEE Trans. Wireless Commun., vol. 14, no. 11, pp. 6164-6176, Nov. 2015.

[15] H. Ma, B. Wang, and K. J. R. Liu, "Distributed signal compressive quantization and parallel interference cancellation for cloud radio access network," IEEE Trans. Commun., vol. 66, no. 9, pp. 4186-4198, 2018.

[16] M. K. Karakayali, G. J. Foschini, and R. A. Valenzuela, "Network coordination for spectrally efficient communications in cellular systems," IEEE Wireless Commun. Mag., vol. 3, no. 14, pp. 56- 61, Aug. 2006.

[17] S. Venkatesan, A. Lozano, and R. Valenzuela, "Network MIMO: Overcoming intercell interference in indoor wireless systems," in Asilomar Conf. on Signals, Systems and Computers, 2007, pp. 83-87.

[18] S. Venkatesan, H. Huang, A. Lozano, and R. Valenzuela, "A WiMAXbased implementation of network MIMO for indoor wireless systems," EURASIP J. on Adv. in Signal Processing, vol. 2009, p. 9, 2009.

[19] D. Gesbert, S. Hanly, H. Huang, S. Shamai, O. Simeone, and W. Yu, "Multi-cell MIMO cooperative networks: A new look at interference," IEEE J. Sel. Areas Commun., vol. 28, no. 9, pp. 1380-1408, 2010.

[20] H. Q. Ngo, A. Ashikhmin, H. Yang, E. G. Larsson, and T. L. Marzetta, "Cell-free massive MIMO versus small cells," IEEE Trans. on Wireless Communications, vol. 16, no. 3, pp. 1834-1850, 2017.

[21] E. Nayebi, A. Ashikhmin, T. L. Marzetta, H. Yang, and B. D. Rao, "Precoding and power optimization in cell-free massive MIMO systems," IEEE Trans. Wireless Commun., vol. 16, no. 7, pp. 4445-4459, 2017.

[22] Z. Chen and E. Björnson, "Channel hardening and favorable propagation in cell-free massive MIMO with stochastic geometry," IEEE Trans. Commun., vol. 66, no. 11, pp. 5205-5219, 2018.

[23] T. C. Mai, H. Q. Ngo, M. Egan, and T. Q. Duong, "Pilot power control for cell-free massive MIMO," IEEE Trans. Vehic. Techn., vol. 67, no. 11, pp. 11264-11268, 2018.

[24] M. Bashar, K. Cumanan, A. G. Burr, M. Debbah, and H. Q. Ngo, "On the uplink max-min SINR of cell-free massive MIMO systems," IEEE Trans. Wireless Commun., vol. 18, no. 4, pp. 2021-2036, Apr. 2019.

[25] G. Interdonato, P. Frenger, and E. G. Larsson, "Scalability aspects of cell-free massive MIMO," in IEEE Int'l Conf. Commun. (ICC'19), May 2019.

[26] S. Buzzi and C. D'Andrea, "Cell-free massive MIMO: User-centric approach," IEEE Wireless Commun. Letters, vol. 6, no. 6, pp. 706-709, Dec. 2017.
[27] M. Attarifar, A. Abbasfar, and A. Lozano, "Modified conjugate beamforming for cell-free massive MIMO," IEEE Wireless Commun. Letters, vol. 8, no. 2, pp. 616-619, Apr. 2019

[28] J. Zhang, S. Chen, Y. Lin, J. Zheng, B. Ai, and L. Hanzo, "Cell-free massive MIMO: A new next-generation paradigm," IEEE Access, vol. 7, pp. 99 878-99888, 2019.

[29] S. Perlman and A. Forenza, "An introduction to pCell," Artemis Networks LLC, White paper, Tech. Rep., Feb. 2015. [Online]. Available: http://www.rearden.com/artemis/ An-Introduction-to-pCell-White-Paper-150224.pdf

[30] M. Attarifar, A. Abbasfar, and A. Lozano, "Random vs structured pilot assignment in cell-free massive MIMO wireless networks," in IEEE Int'l Conf. Commun. Workshops (ICCW'18), 2018, pp. 1-6.

[31] O. Y. Bursalioglu, C. Wang, H. Papadopoulos, and G. Caire, "A novel alternative to cloud RAN for throughput densification: Coded pilots and fast user-packet scheduling at remote radio heads," in Asilomar Conference on Signals, Systems and Computers, Nov. 2016, pp. 3-10.

[32] R. Nikbakht and A. Lozano, "Uplink fractional power control for cellfree wireless networks," IEEE Int'l Conf. Commun. (ICC'19), June 2019.

[33] R. Nikbakht, R. Mosayebi, and A. Lozano, "Uplink fractional power control and downlink power allocation for cell-free networks," IEEE Wireless Commun. Letters, vol. 9, 2020.

[34] R. Nikbakht, A. Jonsson, and A. Lozano, "Unsupervised-learning power control for cell-free wireless systems," in IEEE Int'l Symp. Personal, Indoor and Mobile Radio Commun. (PIMRC'19), Sep. 2019.

[35] — , "Unsupervised-learning power allocation for the cell-free downlink," IEEE Int'l Conf. Commun. Open Workshop on Machine Learning in Communications, June 2020.

[36] S. Buzzi, C. D'Andrea, A. Zappone, and C. D'Elia, "User-centric 5G cellular networks: Resource allocation and comparison with the cellfree massive MIMO approach," IEEE Trans. Wireless Commun., vol. 19, no. 2 , pp. $1250-1264,2020$

[37] J. Morte Palacios, O. Raeesi, A. Gokceoglu, and M. Valkama, "Impact of channel non-reciprocity in cell-free massive MIMO," IEEE Wireless Commun. Letters, vol. 9, no. 3, pp. 344-348, 2020.

[38] E. Nayebi, A. Ashikhmin, T. L. Marzetta, and B. D. Rao, "Performance of cell-free massive MIMO systems with MMSE and LSFD receivers," in Asilomar Conf. on Signals, Systems and Computers, Nov. 2016, pp. 203-207.

[39] E. Björnson and L. Sanguinetti, "Making cell-free massive MIMO competitive with MMSE processing and centralized implementation," IEEE Trans. Wireless Commun., vol. 19, no. 1, pp. 77-90, 2020.

[40] M. Attarifar, A. Abbasfar, and A. Lozano, "Subset MMSE receivers for cell-free networks," IEEE Trans. Wireless Commun., vol. 19, no. 6, pp. 4183-4194, 2020.

[41] P. Liu, K. Luo, D. Chen, and T. Jiang, "Spectral efficiency analysis of cell-free massive MIMO systems with zero-forcing detector," IEEE Trans. Wireless Commun., vol. 19, no. 2, pp. 795-807, 2020.

[42] R. W. Heath Jr and A. Lozano, Foundations of MIMO Communication. Cambridge University Press, 2019.

[43] A. Gersho and T. L. Lim, "Adaptive cancellation of intersymbol interference for data transmission," Bell System Technical Journal, vol. 60 no. 9, pp. 1997-2021, 1981.

[44] M. K. Varanasi and B. Aazhang, "Multistage detection in asynchronous code-division multiple-access communications," IEEE Trans. Commun., vol. 38, no. 4, pp. 509-519, 1990.

[45] H. Elders-Boll, H. D. Schotten, and A. Busboom, "Efficient implementation of linear multiuser detectors for asynchronous CDMA systems by linear interference cancellation," European Trans. Telecommun., vol. 9, no. 5, pp. 427-437, 1998

[46] R. M. Buehrer, S. Nicoloso, and S. Gollamudi, "Linear versus non-linear interference cancellation," J. Commun. Netw., vol. 1, no. 2, pp. 118-133, 1999.

[47] D. Guo, L. Rasmussen, and T. Lim, "Linear parallel interference cancellation in long-code CDMA multiuser detection," IEEE J. Sel. Areas Commun., vol. 17, no. 12, pp. 2074-2081, 1999.

[48] D. Guo, L. Rasmussen, S. Sun, and T. Lim, "A matrix-algebraic approach to linear parallel interference cancellation in CDMA," IEEE Trans. Commun., vol. 48, no. 1, pp. 152-161, 2000.

[49] A. Grant and C. Schlegel, "Iterative implementations for linear multiuser detectors," IEEE Trans. Commun, vol. 49, no. 10, pp. 1824-1834, 2001.

[50] D. R. Brown, M. Motani, V. V. Veeravalli, H. V. Poor, and C. R. Johnson, "On the performance of linear parallel interference cancellation," IEEE Trans. Inform. Theory, vol. 47, no. 5, pp. 1957-1970, 2001.

[51] L. Trichard, J. Evans, and I. B. Collings, "Large system analysis of linear multistage parallel interference cancellation," IEEE Trans. Commun., vol. 50, no. 11, pp. 1778-1786, 2002. 
[52] L. Rasmussen and I. J. Oppermann, "Ping-pong effects in linear parallel interference cancellation for CDMA," IEEE Trans. Wireless Commun., vol. 2, no. 2, pp. 357-363, 2003.

[53] W.-J. Choi, K.-W. Cheong, and J. M. Cioffi, "Iterative soft interference cancellation for multiple antenna systems," in IEEE Wireless Commun. Netw. Conf. (WCNC'00), 2000, pp. 304-309.

[54] Y. Hama and H. Ochiai, "A low-complexity matched filter detector with parallel interference cancellation for massive MIMO systems," in IEEE Int'l Conf. Wireless and Mobile Computing, Networking and Commun. (WiMob'16), Oct. 2016.

[55] P. Som, T. Datta, A. Chockalingam, and B. S. Rajan, "Improved largeMIMO detection based on damped belief propagation," in IEEE Inform. Theory Workshop (ITW'10), 2010.

[56] X. Wang and S. ten Brink, "Iterative MIMO subspace detection based on parallel interference cancellation," in IEEE Wireless Commun. Netw. Conf. (WCNC'17), 2017.

[57] Z. Luo, M. Zhao, S. Liu, and Y. Liu, "Generalized parallel interference cancellation with near-optimal detection performance," IEEE Trans. Signal Proc., vol. 56, no. 1, pp. 304-312, 2007.

[58] X. Wang and H. V. Poor, "Iterative (turbo) soft interference cancellation and decoding for coded CDMA," IEEE Trans. on Communications, vol. 47, no. 7, pp. 1046-1061, 1999.

[59] B. M. Hochwald and S. Ten Brink, "Achieving near-capacity on a multiple-antenna channel," IEEE Trans. on Communications, vol. 51, no. 3, pp. 389-399, 2003.

[60] C. Studer, S. Fateh, and D. Seethaler, "ASIC implementation of softinput soft-output MIMO detection using MMSE parallel interference cancellation," IEEE J. Solid-State Circuits, vol. 46, no. 7, pp. 17541765,2011

[61] M. Witzke, S. Baro, F. Schreckenbach, and J. Hagenauer, "Iterative detection of MIMO signals with linear detectors," in Asilomar Conf. Signals, Systems and Computers, Nov 2002.

[62] A. Tomasoni, M. Ferrari, D. Gatti, F. Osnato, and S. Bellini, "A low complexity turbo MMSE receiver for W-LAN MIMO systems," in IEEE Int'l Conf. Commun., 2006.

[63] S. Zaidi, O. Ben Smida, S. Affes, U. Vilaipornsawai, L. Zhang, and P. Zhu, "User-centric base-station wireless access virtualization for future 5G networks," IEEE Trans. Commun., vol. 67, no. 7, pp. 51905202, 2019.

[64] S. Govindasamy and I. Bergel, "Uplink performance of multi-antenna cellular networks with co-operative base stations and user-centric clustering," IEEE Trans. Wireless Commun., vol. 17, no. 4, pp. 2703-2717, Apr. 2018.

[65] B. Błaszczyszyn, M. K. Karray, and H. P. Keeler, "Wireless networks appear Poissonian due to strong shadowing," IEEE Trans. on Wireless Communications, vol. 14, no. 8, pp. 4379-4390, Aug. 2015.

[66] H. P. Keeler, N. Ross, and A. Xia, "When do wireless network signals appear poisson?" Bernoulli, vol. 24, no. 3, pp. 1973-1994, 2018.

[67] G. George, R. K. Mungara, A. Lozano, and M. Haenggi, "Ergodic spectral efficiency in MIMO cellular networks," IEEE Trans. on Wireless Communications, vol. 16, no. 5, pp. 2835-2849, 2017.

[68] G. George, A. Lozano, and M. Haenggi, "Massive MIMO forward link analysis for cellular networks," IEEE Trans. Wireless Commun., 2019.

[69] 3GPP TS 36.814, "Further advancements for E-UTRA physical layer aspects (Release 9)," 3GPP, Tech. Rep., Mar. 2017.

[70] Q. Sun, D. C. Cox, A. Lozano, and H. C. Huang, "Training-based channel estimation for continuous flat fading BLAST," in IEEE Int'l Conf. Commun. (ICC'02), 2002.

[71] Z. D. Lei and T. J. Lim, "Simplified polynomial-expansion linear detectors for DS-CDMA systems," Electronics Letters, vol. 34, no. 16, pp. 1561-1563, 1998

[72] N. Le Josse, C. Laot, and K. Amis, "Efficient series expansion for matrix inversion with application to MMSE equalization," IEEE Commun. Letters, vol. 12, no. 1, pp. 35-37, 2008.

73] A. Lozano and N. Jindal, "Are yesterday's information-theoretic fading models and performance metrics adequate for the analysis of today's wireless systems?" IEEE Communications Magazine, vol. 50, no. 11, pp. 210-217, Nov. 2012.

[74] R. Nikbakht, A. Jonsson, and A. Lozano, "Unsupervised learning for parametric optimization," IEEE Commun. Letters, vol. 24, 2020.

[75] _ _ "Unsupervised learning for cell-free power control," IEEE Commun. Letters, vol. 24, 2020.

[76] J. F. Whitehead, "Signal-level-based dynamic power control for cochannel interference management," IEEE Veh. Techn. Conf. (VTC'93), pp. 499-502, 1993.
[77] R. D. Yates, "A framework for uplink power control in cellular radio systems," IEEE J. Sel. Areas Commun., vol. 13, no. 7, pp. 1341-1347, 1995.

[78] A. Simonsson and A. Furuskar, "Uplink power control in LTEoverview and performance," IEEE Veh. Techn. Conf. (VTC'08 Fall), pp. 1-5, Sep. 2008.

[79] O. Y. Bursalioglu, C. Wang, H. Papadopoulos, and G. Caire, "RRH based massive MIMO with 'on the fly' pilot contamination control," in IEEE Int'l Conf. on Communications (ICC'16), 2016, pp. 1-7.

[80] Y. Zhang, H. Cao, P. Zhong, C. Qi, and L. Yang, "Location-based greedy pilot assignment for cell-free massive MIMO systems," in IEEE Int'l Conf. on Computer and Commun. (ICCC'18), Dec. 2018, pp. 392-396.

[81] W. Hmida, V. Meghdadi, A. Bouallegue, and J. P. Cances, "Graph coloring based pilot reuse among interfering users in cell-free massive MIMO," IEEE Int'l Conf. Commun. (ICC'20), June 2020.

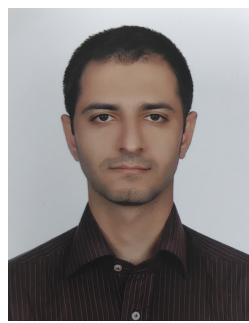

Reza Mosayebi (M'20) received the B.Sc., M.Sc., and Ph.D. degrees (Hons.) in electrical engineering from Sharif University of Technology, Tehran, Iran, in 2012, 2014, and 2018, respectively. In 2017, he was a Visiting Research Scholar with the Friedrich-Alexander University of Erlangen-Nürnberg, Erlangen, Germany. He is currently a Postdoctoral Researcher with Universitat Pompeu Fabra (UPF), Barcelona, Spain. His research interests include wireless and molecular communications, signal processing, and machine learning.

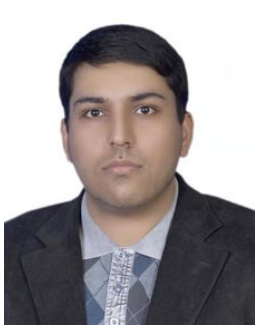

Mohammad Mahdi Mojahedian is currently a Postdoc researcher at Universitat Pompeu Fabra (UPF), Barcelona, Spain. He received two B.Sc. degrees, one in Communications and the other in Electronics, from Amirkabir University of technology (Tehran-Polytechnic), Tehran, Iran, in 2009 and 2010, a M.Sc. degree in Communication Systems from Sharif University of Technology, Tehran, Iran, in 2012, and a PhD. at communication Systems group from Sharif University of Technology, Tehran, Iran, in 2018. Since 2012, he has been with the Information Systems and Security Lab (ISSL) at Sharif University of Technology, and a visiting student at Institute for Digital Communications, University of Erlangen, Nuremberg, from June 2016 to December 2016. He also has been a finalist for the Jack Keil Wolf student paper award, at the ISIT symposium 2015. His research interests include information theory, wireless communications and computer science.

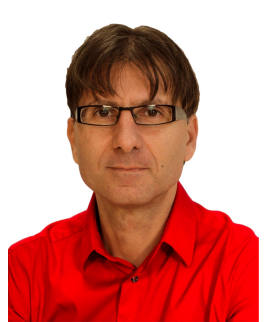

Angel Lozano (S'90-M'99-SM'01-F'14) received his Ph.D. from Stanford University in 1998. In 1999, he joined Bell Labs (Lucent Technologies, now Nokia) in Holmdel, NJ, where he was with the Wireless Communications Research Department until 2008. He is currently a Professor at Universitat Pompeu Fabra (UPF), Barcelona, and the co-author of the textbook "Foundations of MIMO Communication" (Cambridge University Press, 2019). He serves as Area Editor for the IEEE Transactions on Wireless Communications and as Editor for the IEEE Communication Technology News. He received the 2009 Stephen O. Rice Prize for the Best Paper published in the IEEE Transactions on Communications, the 2016 Fred W. Ellersick prize to the best paper published in the IEEE Communications Magazine, and the 2016 Communications Society \& Information Theory Society joint paper award. He holds an Advanced Grant from the European Research Council and was a 2017 Clarivate Analytics Highly Cited Researcher. 